\title{
Die Verfassung der Republik China (Taiwan)
}

\author{
Von Mathias Neukirchen, Berlin ${ }^{*}$
}

\section{Einleitung}

Die Verfassung, vor allem die Verfassungspraxis der Republik China (Taiwan), ist maßgeblich von der seit 1949 bestehenden Teilung Chinas und dem Verhältnis Taiwans zur Volksrepublik China geprägt. Zum besseren Verständnis werden daher erläutert zunächst der Hintergrund der Teilung Chinas (a), die Konkurrenz der Regierungen in Taipei und Beijing um die Vertretung Chinas (b), der völkerrechtliche Status Taiwans (c) und die militärischen Drohungen der Volksrepublik gegenüber Taiwan (d). Anschließend geht es um die Verfassung Taiwans im Kontext dieses Konfliktes (e) und die Ein-China-Politik als Determinante der verfassungsrechtlichen Entwicklung (f).

\section{a. Hintergrund: Die Teilung Chinas nach dem Bürgerkrieg 1949}

Das Ende des chinesischen Bürgerkriegs ${ }^{1} 1949$ teilte China in zwei Herrschaftsbereiche. Das gesamte chinesische Festland wird von der kommunistischen Regierung der im Oktober 1949 proklamierten Volksrepublik China regiert. Die im Bürgerkrieg unterlegene Seite, die zuvor international als Regierung Chinas anerkannte sog. Nationalregierung, floh 1949 vor der siegreichen Roten Armee Maos auf die Inselgruppe Taiwan ${ }^{2}$, welche sie seither ausschließlich regiert. Sie bezeichnet sich weiterhin als Regierung der 1911 proklamierten "Republik China".

Die Ausführungen geben ausschließlich die private Meinung des Autors wieder. Besonderer Dank gebührt Frau Barbara S. Meincke für das Korrekturlesen.

1 Zum Bürgerkrieg und der folgenden Teilung Chinas siehe Weggel, Die Geschichte Taiwans (1991), S. 24ff.; Fairbank, The Great Chinese Revolution 1800 - 1985 (1991), S. $170 \mathrm{ff}$. Penghu $(127 \mathrm{qkm})$ und Jinmen $(150 \mathrm{qkm})$, der Insel Mazu $(29 \mathrm{qkm})$ sowie weiteren 76 kleineren Inseln. 


\section{b. Die Konkurrenz um die Vertretungsbefugnis für China}

Nach dem Ende des Bürgerkrieges 1949 beanspruchte sowohl die Regierung in Beijing als auch die in Taipei, den Staat China bilateral und multilateral, insbesondere in den Vereinten Nationen ${ }^{3}$, zu vertreten. Mehr als zwei Jahrzehnte war die Frage, welche der beiden konkurrierenden Regierungen vertretungsbefugt sei, politisch und völkerrechtlich hoch umstritten ${ }^{4}$. Bis 1971 wurde die Regierung in Taipei von den meisten Staaten und auch den Vereinten Nationen als vertretungsbefugt für China anerkannt, obwohl Taipei tatsächlich über weniger als $1 \%$ der Fläche und Bevölkerung Chinas herrschte ${ }^{5}$. Mit Resolution 2758 vom 26. Oktober 1971 hat die Generalversammlung der Vereinten Nationen dann jedoch in einem Richtungswechsel die Regierung in Beijing als vertretungsbefugt für ganz China anerkannt, auch wenn diese wiederum Taiwan nie beherrscht hat ${ }^{6}$. Parallel zur Entscheidung der Vereinten Nationen anerkannte die große Mehrzahl der Staaten in den bilateralen Beziehungen die Regierung in Beijing als vertretungsbefugte Regierung Chinas und nahm

Die Mitgliedschaft in den Vereinten Nationen ist einem Staat zugeordnet und nicht einer Regierung. Regierungswechsel oder Revolutionen beeinflussen daher die Mitgliedschaft eines Staates in den Vereinten Nationen nicht. Die Mitgliedschaft eines Staates endet nur durch den erklärten Austritt oder Ausschluss des Staates aus den Vereinten Nationen oder durch den Untergang des Staates. Es handelte sich daher im Falle Chinas um eine Frage der Vertretungsbefugnis und nicht der Mitgliedschaft, vgl. hierzu: Neukirchen in VN Vol. 53 (2004), S. 50-55; Ginther in BDGV Vol. 17 (1975), S. 7, 15; Fastenrath, in: Simma, Charta der Vereinten Nationen (1991), Art. 3 Rdnr. 8; Epping, in: Ipsen, Völkerrecht (1999), § 28 Rdnr. 23; Green, CYIL Vol. 10 (1972), S. 102, 103ff.; Schick, ICLQ, Vol. 12 (1963), S. 1232, 1238; Kelsen, The Law of the United Nations (1950), S. 949; Guradze, JZ Vol. 7 (1952), S. 214, 214; v. Perfall, Die völkerrechtliche Vertretung Chinas, Diss. (1967), S. 159.

Siehe die Nachweise bei Neukirchen, Die Vertretung Chinas und der Status Taiwans im Völkerrecht (2004), S. 108ff.

Die Charta der Vereinten Nationen enthält keine Regelung darüber, welche Voraussetzungen eine Regierung erfüllen muss, um einen Mitgliedstaat vertreten zu können. Auch die Geschäftsordnungen der Organe der Vereinten Nationen schreiben nicht vor, wie eine vertretungsbefugte Regierung beschaffen sein muss, vgl. Talmon in Volger, Lexikon der Vereinten Nationen (2000), S. 393, 396; Schwenk, Die Vertretung Chinas in den Vereinten Nationen als völkerrechtliches Problem, Diss. (1959), S. 21; Schermers in EPIL Vol. 2, S. 1320-1324. Lediglich die Prüfung der Vertretungsbefugnis der Regierungsvertreter im Beglaubigungsausschuss (Credential Committee) der Vereinten Nationen ist geregelt, vgl. König, AVR Vol. 28 (1990), S. 266, 270. Bei der Überprüfung der Vollmachten im Beglaubigungsausschuss wird jedoch lediglich geprüft, ob die Delegierten tatsächlich von ihrer Regierung legitimiert sind, und nicht, ob deren Regierung legitimiert ist.

6 Taiwan wurde also nicht aus den Vereinten Nationen ausgeschlossen, es wurde lediglich der Regierung in Taipei die Vertretungsbefugnis für den Mitgliedstaat China aberkannt, siehe dazu: UN-YB 1971, S. 124, 132; Leichter, VN Vol. 19 (1971), S. 149, 151ff.; Neukirchen, a.a.O., S. 107-170; Epping, in: Ipsen, a.a.O., § 28 Rdnr. 23. Es bleibt anzumerken, dass völkerrechtlich betrachtet bereits ab 1950 die Regierung in Beijing vertretungsbefugt war, auch wenn dies in der Praxis erst 1971 anerkannt wurde, siehe hierzu Neukirchen, a.a.O., S. 108ff. 
mit dieser diplomatische Beziehungen auf ${ }^{7}$. Dabei stellten die Völkerrechtssubjekte ebenso wie die Vereinten Nationen klar, Taiwan als Teil der Volksrepublik zu betrachten ${ }^{8}$.

$c$.

Der völkerrechtliche Status Taiwans

Vor dem Hintergrund der vollständigen Unabhängigkeit der Regierung Taiwans von der Regierung in Beijing könnte man von der Existenz zweier chinesischer Staaten, einem Staat "Volksrepublik China" und einem Staat "Taiwan", sprechen. Völkerrechtlich betrachtet kann ein zweiter chinesischer Staat nur durch eine Dismembration des bestehenden Staates oder eine Separation eines Teiles vom bestehenden Staat entstanden sein. Bei der Dismembration zerfällt der alte Staat und es entstehen mindestens zwei neue Staaten; bei der Separation besteht der alte Staat auf einem verminderten Staatsterritorium fort, und es entsteht ein neuer Staat durch Abspaltung.

Eine Dismembration ${ }^{9}$ Chinas in Folge der bürgerkriegsbedingten Teilung Chinas 1949 ist rechtlich jedoch ausgeschlossen, da nahezu das ganze alte Staatsgebiet und der Großteil des Staatsvolkes weiterhin von einer staatlichen Autorität, der Regierung in Beijing, kontrolliert werden ${ }^{10}$. Betrachtet man Festlandchina und Taiwan zusammen als China, so umfasst die Volksrepublik mehr als $99 \%$ der Staatsfläche und Bevölkerung ${ }^{11}$. Sie ist somit geographisch und personell im Wesentlichen identisch mit dem vorher bestehenden Staat China. Eine Dismembration ist also ausgeschlossen und kann nicht zur Entstehung eines neuen, unabhängigen Staates Taiwan geführt haben.

Bislang ist auch kein neuer Staat durch Abspaltung (Separation) Taiwans entstanden. Dies ist ausgeschlossen, da die Regierung in Taipei an der Ein-China-Doktrin festhält. Dies liegt nicht unbedingt an der gemeinsamen Geschichte, Sprache und Kultur und dem mehr oder

Neukirchen, a.a.O., S. 108ff.

Die Regierung der Volksrepublik China verlangte von allen Staaten, die diplomatische (und wirtschaftliche) Beziehungen mit ihr unterhalten wollten, die Anerkennung, dass Taiwan ein Teil Chinas ist. In der Staatenpraxis haben alle Staaten dies daher auch ausdrücklich anerkannt. In den Kommuniqués zur Aufnahme der diplomatischen Beziehungen erfuhr diese Anerkennung dann zahlreiche semantische Variationen. Mit welchen Formulierungen die Staaten gegenüber der Regierung der Volksrepublik die Zugehörigkeit Taiwans zu China anerkannt haben, wird ausführlich dargestellt bei: Fabritzek, Osteuropa Vol. 22 (1972), S. 56, 57ff.; Chen, Soochow Law Review Vol. 7 (1992), S. 301, $321 \mathrm{ff}$.

Zur Dismembration siehe Verdross/Simma, UniversellesVölkerrecht (1984), § 959; Schloh in EPIL Vol. 1, S. 1083, 1085 m.w.N..

10 Neukirchen, a.a.O., S. 77-106; Schick, ICLQ Vol. 12 (1963), S. 1232ff.; v. Perfall, a.a.O., S. 157f.; Dagegen spricht auch, dass Beijing und Taipei eine Dismembration ausschließen. 
minder klaren kulturellen Zusammengehörigkeitsgefühl der Menschen auf beiden Seiten der Taiwan-Straße, die sich alle als Chinesen bezeichnen. Dies liegt - zumindest seit den letzten 20 Jahren - wohl eher an der militärischen Drohung der Regierung der Volksrepublik China gegenüber der Regierung in Taipei, im Falle einer Abkehr von der Ein-ChinaDoktrin, die Einheit Chinas militärisch wiederherzustellen. Trotz der großen politischen, verfassungsrechtlichen und außenpolitischen Reformen hat Taiwan zu keinem Zeitpunkt die Unabhängigkeit erklärt. Ebenso wenig dokumentiert Taiwan in der neuen "pragmatischen Außenpolitik"12 ein eigenständiges Staatsverständnis, da auch hierbei trotz aller Reformen offiziell an der Einheit Chinas festgehalten wird.

Taiwan fehlt daher weiterhin ein eigenes, von China unabhängiges, neues staatliches Selbstverständnis. Der Manifestation eines eigenen Staatsverständnisses steht die von Taipei vertretene "Ein-China-Politik" entgegen ${ }^{13}$. Politisch verständlich, völkerrechtlich aber nicht beachtlich ist, dass Taiwan aufgrund einer möglichen militärischen Reaktion der Volksrepublik China vor einer klaren Artikulation eines eigenstaatlichen Selbstverständnisses zurückschreckt ${ }^{14}$. Für die Entstehung eines neuen (taiwanesischen) Staates ist ein solches Selbstverständnis jedoch Voraussetzung ${ }^{15}$.

Einem Regime, das sich selbst nicht als eigener, neuer Staat begreift, darf aber nicht die Staatseigenschaft von außen oktroyiert werden. Solange Taiwan sich nicht als von China

12

13

Die Regierung der Volksrepublik betont dies im "Chinese Whitepaper on Cross-Strait Relations" vom August 1993 (http://www.chinataiwan.org/web/webportal/W5023254/index.html), und die Regierung der Republik China im "Taiwanese White Paper on Cross-Strait Relations" vom Juli 1994 (http://www.mac.gov.tw/english/index1-e.html); Siehe hierzu: Chen, PILJ Vol. 4 (1965), S. 99, 147ff.; Clough, CYILA Vol. 1 (1981), S. 17, 17f. Beide Texte finden sich auch bei Henckaerts, (Hrsg.), The international status of Taiwan in the new world order (1996), S. 267292.

14

15

Sandschneider, Außenpolitik Vol. 43 (1990), S. 92, 100; Munro, Foreign Affairs Vol. 73 (1994) Nr. 6, S. 109, 117.

Mit derselben Begründung wird Taiwan auch von anderen Stimmen in der Literatur die Staatseigenschaft nicht zuerkannt: Talmon, Recognition of Governments in International Law (1998), S. 299; Roeper, Geteiltes China: eine völkerrechtliche Studie (1968), S. 203; Verdross/Simma, a.a.O., § 387; Heuser in EPIL Vol. 3, S. 753, 757; Dugard, Recognition and the United Nations (1976), S. 71; Ress, Dokumente und Schriften der Europäischen Akademie Otzenhausen Nr. 86 (1995), S. 63, 72ff.; Chen, PILJ Vol. 4 (1965), S. 99, 140ff.; Crawford, The Creation of States in International Law (1979), S. 151f.; Manin, AFDI Vol. 26 (1980), S. 141, 152; Breytenbach, SAJIL Vol. 2 (1994), S. 50, 52; Wright, AJIL Vol. 49 (1955), S. 320, 336; O'Connell, AJIL Vol. 50 (1956), S. 405, 415; Shaw, International Law (1991), S. 162 f.; Ein subjektives Element der Identitätsbehauptung ist erforderlich, vgl. Ipsen, Kultur und Politik Vol. 1 (1975), S. 2, 15ff. Döhring konstatiert allgemein, dass schon kein Staatsvolk vorhanden ist, wenn dieses nicht mehrheitlich den Willen hat, als eigener Staat zusammengefasst zu sein, Doehring, EPIL Vol. 4, S. 600, 601f. Anderer Ansicht ist beispielweise Wachmann, JEAA Vol. 35 (2000), S. 183, 198ff. 
unabhängiger Staat betrachtet, stellt es völkerrechtlich lediglich ein stabilisiertes de facto Regime und nicht einen eigenen Staat dar ${ }^{16}$. Da es weder zu einem Zerfall Chinas in mehrere Nachfolgestaaten (Dismembration) noch zu einer Separation Taiwans von China gekommen ist ${ }^{17}$, existiert völkerrechtlich nur ein chinesischer Staat (die Volksrepublik China) und ein de facto Regime (Taiwan). Dem entspricht auch, dass - im Gegensatz zur Volksrepublik - Taiwan weder von den Vereinten Nationen noch von anderen Staaten als (neu entstandener) Staat anerkannt wird, sondern als Teil der Volksrepublik betrachtet wird $^{18}$. Nur eine Minderheit von gegenwärtig 26 Staaten unterhält formelle diplomatische Beziehungen zu Taiwan ${ }^{19}$. Im Unterschied zu anderen geteilten Staaten (z.B. Korea, Jemen und Deutschland) sagte sich mindestens einer der beiden Teile von dem gemeinsamen Staat los, und es kam zur Dismembration oder Separation und anschließend über kurz oder lang zur Anerkennung von zwei Staaten ${ }^{20}$.

\section{d. Die militärischen Drohungen der Volksrepublik China gegenüber Taiwan}

Beijing hält fest an seinem Rechtsstandpunkt, Taiwan sei eine Provinz der Volksrepublik. Seit mehr als 30 Jahren droht Beijing, die Einheit Chinas militärisch wiederherzustellen, wenn Taiwan die Wiedervereinigung langfristig verschleppt oder sich für unabhängig

Vgl.: Breytenbach, SAJIL Vol. 2 (1994), S. 50, 52; Charney/Prescott, AJIL Vol. 94 (2000), S. 453, 468ff.; Chiang, Fordham International Law Journal Vol. 23 (2000), S. 959, 980ff; Clough, Proc. ASIL Vol. 87 (1993), S. 73, 73f.; Frowein in EPIL Vol. 1, S. 966, 966; Heuser in EPIL Vol. 4, S. 753, 753ff.; Hsiao, New England Law Review Vol. 32 (1998) Nr. 3, S. 1, 8ff.; Li, Northwestern Journal of International Law and Business Vol. 1 (1979), S. 134, 138ff.; Manin, AFDI Vol. 26 (1980), S. 141, 143; Neukirchen, a.a.O., S. 217ff; Roeper, a.a.O., S. 98ff.; Shaw, a.a.O., S. 162 f.; Shen, American University International Law Review Vol. 15 (2000), S. 1101, 1139; van Vranken Hickey, JEAA Vol. 7 (1993), S. 563, 585f.; Verdross/Simma, a.a.O., § 387; Weng, The China Quarterly Vol. 99 (1984), S. 462, 478; Yu, VN Vol. 19 (1971), S. 98, 99f. Anderer Ansicht sind: Caty, Le statut juridique des états divisés (1969), S. 29f.; Chen, Soochow Law Review Vol. 7 (1992), S. 301, 301ff.; Chiu, Contemporary China Vol. 112 (1992) Nr. 5, S. 1, 24ff.; Feldman, Taiwan and the United Nations (1995), S. 1ff.; Chang/Lim, Journal of International Law and Foreign Affairs Vol. 1 (1996) Nr. 2, S. 393, 403ff.; Chen, New England Law Review Vol. 32 (1998) Nr. 3, S. 1, 1f.; Dean, Proc. ASIL Vol. 49 (1955), S. 86, 94; Klintworth, New Taiwan, New China (1995), S. 260f.; Schick, ICLQ Vol. 12 (1963), S. 1232, 1238; v. Perfall, a.a.O., S. 157f.

Vgl. hierzu ausführlich Neukirchen, a.a.O. , S. 263-314.

Siehe hierzu Neukirchen, a.a.O., S. 217-242.

19

20 Stand 1.10.2005, siehe http://www.gio.gov.tw/taiwan-website/5-gp/yearbook/P113.htm\#3

Zum Vergleich mit Deutschland siehe: Domes, Issues \& Studies Vol. 28 (1992) Nr. 2, S. 11-27; Garver, JEAA Vol. 8 (1994) Nr. 1, S. 135-172; Kindermann, Issues \& Studies Vol. 29 (1993) Nr. 5, S. 39-69; Möller, The China Quarterly Nr. 147 (1996), S. 706-725. 
erklären sollte. Dies ist mehrfach offiziell, z.B. im Weißbuch von 1993 zu den Beziehungen in der Taiwanstraße, bekundet worden ${ }^{21}$.

Auf der Plenarsitzung des Nationalen Volkskongresses am 14. März 2005 haben diese Drohungen ein neues Niveau erreicht: Dort wurden ohne Gegenstimme nicht nur die Militärausgaben um 12,6\% angehoben, sondern auch das sog. Anti-Sezessionsgesetz verabschiedet $^{22}$. Dies ermächtigt den Staatsrat und die Militärkommission mit Staats- und Parteichef Hu Jintao an der Spitze, im Falle einer Unabhängigkeitserklärung Taiwans „oder anderer größerer Zwischenfälle" militärisch gegen Taiwan vorzugehen. Es handelt sich de facto um ein Kriegsermächtigungsgesetz, welches militärische Maßnahmen gegen Taiwan vorab innerstaatlich legalisiert.

Das Risiko und die Bedeutung eines solchen Konfliktes sind offensichtlich: In wirtschaftlicher Hinsicht stehen sich in der Taiwanstraße die dritt- (Volksrepublik) und fünfzehntgrößte (Taiwan) Handelsnationen der Welt mit den nach Japan weltweit größten Devisenreserven gegenüber. In militärischer Hinsicht handelt es sich um die beiden seit Jahren größten Rüstungsimporteure. Ein militärischer Konflikt zwischen den beiden hochgerüsteten Seiten ist trotz der fortschreitenden wirtschaftlichen Verflechtung nicht weniger wahrscheinlich geworden. Er birgt zudem das Risiko einer Internationalisierung. Die USA haben sich im Taiwan Relations Act zu militärischen Lieferungen zur Verteidigung Taiwans verpflichtet und darüber hinaus 1996 während der sog. Raketenkrise die 7. US-Flotte zum Schutz Taiwans in die Taiwanstraße kommandiert und damit gezeigt, wie ernst sie ihre Verbürgungen gegenüber Taiwan nehmen ${ }^{23}$. Sind die militärischen Drohungen der Volksrepublik China gegenüber Taiwan völkerrechtskonform ?

Als 1996 Raketen in taiwanesischen Hoheitsgewässern einschlugen, erklärte der damalige UN-Generalsekretär Boutros Boutros-Ghali, Taiwan sei eine Provinz Chinas, es handele sich um eine innerchinesische Angelegenheit ${ }^{24}$. Die meisten Staaten bewerteten dies ähnlich, schon um ihrer diplomatischen (und wirtschaftlichen) Beziehungen zu Beijing

"Chinese Whitepaper on Cross-Strait Relations" vom August 1993 siehe: http://www.chinataiwan. org/web/webportal/W5023254/index.html.

Vgl. Beijing Rundschau 2005-13.

23

Siehe Shambaugh, Spiegel Special 5/2004, S. 37f. Zur Friedensgefährdung in den letzten 25 Jahren siehe: Chang, Orbis Vol. 40 (1996) Nr. 4, S. 577-607; Freeman, Foreign Affairs Vol. 77 (1998) Nr. 4, S. 6-11; Hu, JEAA Vol. 11 (1997) Nr. 1, S. 149-182; Huang, Issues \& Studies Vol. 28 (1992) Nr. 6, S. 35-58; Kindermann, Internationale Politik Vol. 50 (1995) Nr. 10, S. 35-41; Lin, The China Quarterly Nr. 146 (1996), S. 577-595; Shambaugh, The China Quarterly Nr. 148 (1996), S. 1284-1318; Vranken Hickey, The Pacific Review Vol. 5 (1992) Nr. 3, S. 250-258; Weggel, China Aktuell 2/2000, S. 148-154; Yu, Asian Outlook Vol. 26 (1981) Nr. 2, S. 19-23.

UN-Doc. SG/SM/5919 vom 8.3.1996. 
willen. Die UN wie die Mehrheit der Staatengemeinschaft verzichteten darauf, diese Drohungen völkerrechtlich zu verurteilen.

Völkerrechtlich betrachtet muss man jedoch zu einem anderen Ergebnis kommen: Das Antisezessions-Gesetz stellt völkerrechtlich unzweifelhaft eine Drohung mit Gewalt dar. Das Gewaltverbot der Charta der Vereinten Nationen gilt auch gegenüber stabilisierten de facto Regimen ${ }^{25}$. Taiwan ist ein stabilisiertes de facto Regime, so dass die militärischen Drohungen Beijings völkerrechtswidrig sind. Das EU-Waffenembargo gegenüber der Volksrepublik aufzuheben, wäre in dieser Situation sicherlich ein falsches Zeichen.

\section{e. Die Verfassung Taiwans im Kontext des Konfliktes in der Taiwanstraße}

Die seit 1947 in Taiwan formell unverändert fortgeltende Verfassung geht von der Einheit Chinas in den damaligen Grenzen aus ${ }^{26}$. Sie wurde nie geändert, um auch verfassungsrechtlich an der Einheit Chinas festzuhalten. Eine Reform der Verfassung könnte von der Volksrepublik als eine Abkehr von der Ein-China-Doktrin gewertet werden. Für diesen Fall hat die Volksrepublik mehrfach und unmissverständlich klargestellt, dass sie dann die Einheit Chinas militärisch wiederherstellen werde. Verfassungsänderungen in Taiwan haben daher eine besondere Bedeutung.

\section{f. Die Ein-China-Doktrin als Determinante der verfassungsrechtlichen Entwicklung}

An der Ein-China-Doktrin hält nicht nur die Volksrepublik, sondern auch Taiwan bis heute verfassungsrechtlich fest. Die Ursachen für das jahrzehntelange Festhalten Taiwans an der Einheit Chinas änderten sich im Laufe der Zeit. Noch In den fünfziger Jahren hoffte die Regierung in Taipei auf innenpolitische Schwierigkeiten der Kommunisten und die "glorreiche Rückeroberung" des chinesischen Festlandes. In den sechziger Jahren hielt Taiwan an der Einheit Chinas fest, um die Volksrepublik und die Regierung in Beijing durch eine der "Hallstein-Doktrin" ähnliche Außenpolitik zu isolieren". Als Anfang der siebziger Jahre die weltweite Anerkennung der Regierung in Beijing nicht mehr aufzuhalten war,

Crawford, a.a.O., S. 107 und 152; Hsiao, New England Law Review Vol. 32 (1998), S. 1, 5; Frowein EPIL Vol. 2, S. 966, 966; Verdross/Simma, a.a.O. , § 406; Frowein, Das de facto Regime im Völkerrecht (1968), S. 35ff. mit weiteren Nachweisen.

Schubert, Rückblick auf eine Dekade des demokratischen Wandels- Reformen in Politik und Gesellschaft Taiwans, 1986-1994 (1995), S. 17, 27; Chiang, Fordham International Law Journal Vol. 23 (2000), S. 959, 985.

Clough, CYILA Vol. 1 (1981), S. 17, 17f.; Weng, The China Quarterly Vol. 99 (1984), S. 462, 464. Zur Hallsteindoktrin siehe Kilian, Die Hallstein-Doktrin: der diplomatische Krieg zwischen der BRD und der DDR 1955-1973 (2001). 
verkehrte sich die Wirkung in das Gegenteil: Isoliert war nunmehr die Regierung in Taipei. Autorität geführt unter Staatspräsident General Chiang Kai-shek hielt sie jedoch an der EinChina-Politik fest und versuchte, durch den Kampf gegen die Regierung in Beijing innenpolitische Beschränkungen und Demokratiedefizite zu legitimieren.

Auch nach der Demokratisierung Taiwans Ende der achtziger Jahre und in den neunziger Jahren ${ }^{28}$ hielt die neue Regierung unter Präsident Li an der Ein-China-Doktrin fest ${ }^{29}$. Auch der seit Juni 2000 amtierende und bis 2008 gewählte Präsident Chen Shui-bian will die Ein-China-Doktrin nicht aufgeben ${ }^{30}$.

\section{Die Entstehungsgeschichte der Verfassung von 1947}

Die noch heute in Taiwan gültige "Verfassung der Republik China" wurde vor der Teilung Chinas erarbeitet und daher für Gesamtchina konzipiert. Sie trat 1947 in Kraft. Ihr geistiger Vater war Dr. Sun Yatsen, auf beiden Seiten der Taiwanstraße als „Gründer des modernen Chinas" verehrt.

a. Die Lehren Sun Yatsens zur Verfassung

Als der Arzt und charismatische Führer Dr. Sun Yatsen sich 1924 Gedanken über die Zukunft Chinas machte, stand das Reich der Mitte kurz vor dem Kollaps: Die imperialistischen Staaten hatten immer größere Teile Chinas als Pachtgebiete erpresst und immer weitergehende Sonderrechte erhalten. Im Inneren war China seit dem Sturz des Kaisers und

Nach dem Tod von Chiang Kaishek (1975) übernahm dessen Sohn Jiang Jingguo die Führung des Staates. Er ordnete am 14. Juli 1987 die Aufhebung des Kriegsrechts in Taiwan an, das über Jahrzehnte die Grundrechte inklusive der Meinungs- und Pressefreiheit stark eingeschränkt hatte. Nach Jiang Jingguos Tod 1988 wurde 1990 Li Denghui neuer Präsident. Er demokratisierte Taiwan, ließ Oppositionsparteien zu und führte freie Wahlen durch. Zur Nachfolge als Staatspräsident und Parteichef der GMD, China aktuell 8/1990, S. 647.

29

Zur Demokratisierung siehe Schubert, a.a.O., S. 17-29; Domes, Die Herausbildung eines Mehrparteien-Konkurrenzsystems in der Republik China auf Taiwan (1995), S. 29-39; Schubert, Taiwan, die chinesische Alternative. Demokratisierung in einem ostasiatischen Schwellenland (19861993)(1994), S. 1ff.

30 Dies hat Präsident Chen Shuibian, der zugleich bis Dezemebr 2004 Vorsitzende der DFP war, regelmäßig erklärt, zum Beispiel in einem Interview mit dem SPIEGEL Vol. 54 (2000) Nr. 42, S. 265, 265f. Zur Chinapolitik der DFP siehe auch Wang, in Lee (Hrsg.), Taiwan in perspective (2000), S. 159-182; Trampedach in Fabrowsky/Milbrodt (Hrsg.), Taiwan nach den Präsidentschaftswahlen vom März 2000 (2000), S. 9-26; Weggel in Fabrowsky/Milbrodt, a.a.O., S. 41-58; Lijun, in Trends in Southeast Asia Vol. 11 (9/2000) S. 1-38. 
der Proklamation der Republik 1911 von inneren Unruhen und der Herrschaft regionaler Warlords geprägt. Die offizielle Regierung hatte über weite Teile des Landes keine Kontrolle und die im März 1912 erlassene "Provisorische Verfassung der Republik China" blieb Makulatur.

Vor diesem Hintergrund formulierte Sun Yatsen seine "Grundlinien für den Aufbau des Staates durch die Nationalregierung" ${ }^{31}$. Er wollte zwar einen Verfassungsstaat, hielt aber die Wiederherstellung der Einheit Chinas und die politische Unterweisung der Bevölkerung für prioritär und sah darin die wesentliche Voraussetzung für den Erfolg einer Verfassung. Nach seiner Lehre sollte daher zunächst in einer "Phase der Militärherrschaft" (junzheng) eine Militärregierung (die Nationalregierung der Guomindang, GMD) mit Waffengewalt China einigen. Nach Sun Yatsen sollte in einer hierauf folgenden "Phase der Herrschaft durch Unterweisung" (xunzheng), das chinesische Volk politisch durch die Nationalregierung unterwiesen werden. Erst in einer dritten Phase sollte dann eine Verfassung erarbeitet und in Kraft gesetzt werden, "Herrschaft kraft Verfassung" (xianzheng). Sun Yatsen erlebte die Verwirklichung seiner Lehren nicht mehr. Er starb 1925.

Wenig später begann die GMD mit General Chiang Kaishek an der Spitze die militärische Wiedervereinigung Chinas, die 1928 gelang. Entsprechend der Lehre Sun Yatsen's wurde zunächst keine Verfassung sondern lediglich das rudimentäre, nur zehn Paragraphen umfassende Organisationsgesetz ${ }^{32}$ der Nationalregierung beschlossen. Hierin wurde - nach russischem Vorbild - die Führungsrolle der GMD festgeschrieben. Auf Druck der Bevölkerung erließ die Nationalregierung 1931 dann eine „Vorverfassung“33, welche aber weder rechtsstaatlichen noch demokratischen Maßstäben genügte ${ }^{34}$ und primär dem Machterhalt der GMD diente. Auch der von der GMD überarbeitete neue Verfassungsentwurf, 1936 von

Das Organisationsgesetz bestimmte u.a., dass die Nationalversammlung zum Großteil nicht gewählt, sondern ex lege aus Mitgliedern der GMD bestehen sollte, die zudem auf die Auswahl der anderen Wahlkandidaten einen erheblichen Einfluss hatten, Lasars, JöR n.F Vol. 41 (1993), S. 597, 616. Eine deutsche Übersetzung des Organisationsgesetz der Nationalregierung vom 4. Oktober 1928, findet sich in ZaöRV Vol. 1 (1929), S. 487-491.

Die "Vorverfassung der Republik China" vom 1. Juni 1931 findet sich in einer deutschen Übersetzung bei Chang, Die staatsrechtliche Krise der chinesischen Republik, JÖR a.F. Vol. 19 (1931), S. $316,347 \mathrm{ff}$. dass Delegierte der GMD die Nationalversammlung ersetzen und das zentrale Regierungsrecht ausüben, Lasars, JöR n.F. Vol. 41 (1993), S. 597, 610ff. 
der Nationalregierung bekannt gegeben ${ }^{35}$, sicherte verfassungsrechtlich die EinparteienHerrschaft der GMD ${ }^{36}$ und wurde vom Volk abgelehnt.

Die bürgerkriegsähnlichen Auseinandersetzungen mit den Kommunisten (1928-1937) und die großflächige Invasion Chinas durch Japan im Zweiten Weltkrieg (1937-1945) verhinderten in der Folgezeit den Erlass einer neuen Verfassung. Bei Kriegsende galt so weiterhin die Vorverfassung von $1931^{37}$. Erst 1945 konnte daher die von Sun Yatsen vorgesehene dritte Phase der "Herrschaft kraft Verfassung" (xianzheng) beginnen.

Im November 1946 trat eine verfassungsgebende Nationalversammlung zusammen, an der jedoch überwiegend nur Delegierte der GMD teilnehmen durften. Die Kommunistische Partei Chinas (KPCh) boykottierte die Nationalversammlung ${ }^{38}$. Der Krieg zwischen der Nationalregierung und den Kommunisten begann erneut.

Die Nationalversammlung tagte dessen ungeachtet weiter und verabschiedete Ende 1947 die Verfassung, die bis heute in Taiwan gilt ${ }^{39}$. Trotz des Bürgerkrieges fanden 1948 Wahlen für die verfassungsrechtlich vorgesehenen Volksvertretungsorgane statt ${ }^{40}$. Die erste Nationalversammlung trat im März 1948 erstmals zusammen und wählte am 19. April 1948 Chiang Kaishek, den seit 1938 amtierenden Parteivorsitzenden der GMD und Chef der bisherigen Nationalregierung, zum ersten Präsidenten nach der neuen Verfassung ${ }^{41}$. Die am

Der Verfassungsentwurf wurde am 5. Mai veröffentlicht und ging deshalb als "Verfassungsentwurf vom 5. Mai" in die chinesische Verfassungsgeschichte ein, hierzu ausführlich Lasars, JöR n.F. Vol. 41 (1993), S. 597ff.; Heuser, JöR n.F. Vol. 41 (1993), S. 659, 661

Lasars, JöR n.F. Vol. 41 (1993), S. 597, 614; Wu, Streitbare Demokratie, S. 110.

Im Januar 1946 begann nach Vermittlung durch die USA die "Politische Konsultativkonferenz", auf der alle Parteien Chinas, auch GMD und KPCh, eine neue Verfassung erarbeiten sollten. Die ideologischen Differenzen zwischen GMD und KPCh waren aber größer als die Gemeinsamkeiten. Die auf der Konferenz am 31. Januar 1946 verabschiedeten "Prinzipien der Politischen Konsultativkonferenz zur Änderung der Verfassung" traten jedoch aufgrund des beginnenden Bürgerkriegs zwischen GMD und KPCh so nicht in Kraft, siehe hierzu Lasars, JöR n.F. Vol. 41 (1993), S. 597, 614 .

Die neue Verfassung wurde von der Regierung am 25. Dezember 1946 angenommen, am 1. Januar 1947 von der Nationalregierung verkündet und trat am 25. Dezember 1947 in Kraft, AdG, 24.12.1946, 960 J. Diese Verfassung findet sich in einer deutschen Übersetzung in ZaöRV, Vol. 13 (1950/51), S. 816-836, eine englische Übersetzung findet sich JöR n.F. Vol. 27 (1978), S. 564576. organe auf drei Jahre bzw. sechs Jahre gewählt.

AdG, 19.4.1946, 1469 D. 
20. Mai 1948 gebildete neue Regierung ${ }^{42}$, ähnelte personell so sehr der vorherigen, dass man sie weiterhin "Nationalregierung" nannte ${ }^{43}$.

\section{b. Das Konzept der Verfassung der Republik China von 1947}

Das Konzept der Verfassung von 1947 unterscheidet sich von europäischen Verfassungen zum Teil erheblich ${ }^{44}$ und fußt auf der Verfassungslehre Sun Yatsens. Sie proklamiert in Art. 1 "Drei Prinzipien des Volkes" (minzuzhuyi): Nationalismus ${ }^{45}$, Volksherrschaft ${ }^{46}$ und Volkswohlfahrt ${ }^{47}$. Ausgangspunkt ist Suns "Lehre der Unterscheidung von Macht und Fähigkeit", nach der das Volk die Macht und die Regierung die Fähigkeiten besitzt ${ }^{48}$. In diesem Sinne wird zwischen den Gewalten der Regierenden und den Gewalten der Regierten (des Volks) unterschieden.

Das Volk hat vier Gewalten: die Wahl einer Regierung, deren Abwahl, die Gesetzesinitiative und das Referendum. Diese Volksgewalten werden durch die für sechs Jahre gewählte Nationalversammlung ausgeübt. Diese wählt den Präsidenten und ist für Verfassungsänderungen, nicht aber die sonstige Rechtsetzung oder die Kontrolle der Regierung zuständig und tagt daher nicht häufig.

AdG, 1.6.1948, 1515 B.

Chen/Reisman, Yale Law Journal Vol. 81 (1972), S. 599, 613.

Die Weimarer Verfassung übte einen nicht unerheblichen Einfluss auf die Verfassung der Republik China aus, insbesondere der umfangreiche Grundrechtskatalog ähnelt dem der Weimarer Verfassung, Yao, JöR n.F. Vol. 35 (1986), S. 618, 635; Lasars, JöR n.F. Vol. 41 (1993), S. 597, 621. Der Einfluss des deutschen Verfassungsrechts zeigt sich auch daran, dass sehr viele der Obersten Richter Taiwans in Deutschland studiert hatten, in taiwanesischen Verfassungsrechtslehrbüchern deutsche Verfassungen behandelt und sogar Begriffe der deutschen Verfassungslehre und Rechtsprechung des Bundesverfassungsgerichts als Fachtermini auf Deutsch angegeben werden.

Hiermit war ursprünglich die Wiedergewinnung der Herrschaft durch die Han-Bevölkerungsmehrheit im von den Mandschu-Eroberern dominierten Kaiserreich gemeint. Erst nach dem Sturz des Kaiserreichs erhielt der Begriff eine andere Bedeutung: Das Prinzip des Volkstums fordert einen unabhängigen und gleichberechtigten Status für China innerhalb der Staatengemeinschaft und die Gleichheit für alle ethnischen Gruppen innerhalb Chinas.

Das Prinzip der Volksherrschaft bestimmt die individuellen wie die bürgerlichen Freiheiten des Einzelnen.

Das Prinzip der Volkswohlfahrt propagiert eine prosperierende und gerechte Gesellschaft, ein Ziel, das durch eine Verbindung von freiem Unternehmertum mit wirksamer staatlicher Planung erreicht werden soll. 
Der Regierung stehen fünf Gewalten zu: die Gesetzgebung, die Verwaltung, die Rechtsprechung, die Prüfung und die Kontrolle. Die beiden letzteren entspringen der chinesischen Tradition $^{49}$ und gehen auf vergleichbare Institutionen im kaiserlichen China zurück ${ }^{50}$. Jede der fünf Gewalten der Regierung ist in der Verfassung einem Regierungshof (Yuan) zugewiesen $^{51}$ :

- Der Exekutivyuan (Verwaltungshof) gemäß Art. 53 ist die Regierung ${ }^{52}$ der Republik China, wird vom Premierminister geleitet und steht den Ministerien und verschiedenen Kommissionen vor.

- Der Legislativyuan (Gesetzeshof) gemäß Art. 62 fungiert als eine Art Parlament ${ }^{53}$ und verabschiedet die Gesetze.

- Der Justizyuan ${ }^{54}$ (Justizhof) gemäß Art. 77 leitet das Justizwesen und errichtet gemäß Art. 78 und 79 eine Versammlung der Hohen Richter, eine Art Verfassungsgericht ${ }^{55}$.

- Der Prüfungsyuan ${ }^{56}$ (Prüfungshof) gemäß Art. 83 nimmt die Aufgaben der Prüfung sowie Einstellung und Leitung des gesamten im öffentlichen Dienst beschäftigten Personals wahr.

49

50

Yao, JöR n.F. Vol. 35 (1986), S. 618, 621.

Roll, Außenpolitik Vol. 15 (1964), S. 645, 646ff.; Lasars, JöR n.F. Vol. 41 (1993), S. 597, 619.

Bünger, ZaöRV Vol. 13 (1950), S. 759, 780ff. Zu dieser Einteilung der Verfassungsorgane siehe Sun Yatsen, Fundamentals of national Reconstruction (1952).

Im Unterschied zur abendländischen Verfassungstraditionen ist die Exekutive, der Exekutivyuan, nicht für die gesamte Verwaltung zuständig. Die Befugnis in Prüfungs- und Personalangelegenheiten obliegt einer eigenständigen fünften Gewalt, dem Prüfungsyuan.

Nach diesem Verfassungssystem ist der Gesetzeshof nur bedingt mit Parlamenten im europäischen Sinne zu vergleichen: Der Legislativyuan wird vom Volk direkt gewählt und hat zwar die allgemeine Gesetzgebungskompetenz, ihm fehlt aber das Recht zu Verfassungsänderung, das bei der Nationalversammlung liegt. Die Kontrolle der Regierung obliegt nicht dem Legislativyuan, sondern dem Kontrollyuan zusteht.

Der Justizyuan setzt sich aus einem Präsidenten, einem Vizepräsidenten sowie 15 Obersten Richtern zusammen, die alle vom Präsidenten mit Zustimmung der Nationalversammlung ernannt werden. Er ist das höchste Rechtsorgan des Landes. Ihm sind die Institutionen der ordentlichen und der Verwaltungsgerichtsbarkeit und die Disziplinarkommission für Beamte nachgeordnet. Die 15 obersten Richter bilden eine Versammlung der hohen Richter, die für die Interpretation der Verfassung und die Vereinheitlichung von Gesetzesinterpretationen zuständig ist. Die Stellung und Organisation dieser Versammlung der hohen Richter entspricht etwa der des Bundesverfassungsgerichts, Weng, JöR n.F. Vol. 21 (1972), S. 639 (649ff.).

Das Gerichtssystem fällt in die Zuständigkeit des Judikativyuan. In den 20 Distriktgerichten gibt es je eine Straf- eine Zivil- und eine summarische Abteilung, in welcher einfach gelagerte Fälle behandelt werden. Berufungen werden beim Hohen Gericht erhoben, die Revisionen ist beim Höchste Gericht zulässig. Die Versammlung der Hohen Richter ist für die Auslegung der Verfassung zuständig. Zum Justizsystem siehe Chung, in Free China Review Vol. 11 (1998) Nr. 8, S. 14.

Der Prüfungsyuan setzt sich aus einem Präsidenten, einem Vizepräsidenten sowie 19 weiteren Mitgliedern zusammen, die vom Präsidenten für eine sechsjährige Amtszeit ernannt und von der Nationalversammlung bestätigt werden müssen. Der Prüfungsyuan ist für die Prüfungen der Mit- 
- Der Kontrollyuan ${ }^{57}$ (Kontrollhof) gemäß Art. 90 fungiert einerseits wie ein Rechnungshof, andererseits wie ein ständiger parlamentarischer Untersuchungsausschuss und soll die Regierung kontrollieren.

Demokratisch direkt legitimiert sind die Mitglieder des Legislativyuan, des Kontrollyuan und der Nationalversammlung. Der Präsident wird indirekt durch die Nationalversammlung gewählt. Er ernennt mit Zustimmung des Legislativyuan den Regierungschef (Premierminister), den Vorsitzenden des Exekutivyuan ${ }^{58}$, und mit Zustimmung der Nationalversammlung den Präsidenten, den Vizepräsidenten und die Obersten Richter des Justizyuan, sowie den Präsidenten, den Vizepräsidenten und die Mitglieder des Prüfungsyuan. Der Präsident ist zudem für die Verkündung von Gesetzen und den Abschluss völkerrechtlicher Verträge zuständig und führt den Oberbefehl über die Streitkräfte.

Der Vorsitzende des Exekutivyuan, der Premierminister, ist der Regierungschef und die höchste exekutive Macht. Er gehört weder dem Legislativyuan an, noch kann er von ihm gestürzt werden. Der Präsident ist durch die Nationalversammlung demokratisch legitimiert und unabhängig von Legislativyuan und Exekutivyuan. Er verfügt zwar über keine der klassischen exekutiven Kompetenzen, kann aber durch sein Recht, Notverordnungen und das Kriegsrecht zu erlassen, zur "zweiten Exekutive" werden ${ }^{59}$.

Das System der fünf Gewalten hat zu keiner Zeit im Sinne seines Schöpfers funktioniert und war ein problematischer Versuch, Einrichtungen, die mit dem konfuzianischen Staat verbunden waren, in eine moderne Staatsordnung einzufügen ${ }^{60}$. Insgesamt ist dieses Ver-

arbeiter im öffentlichen Dienst, bestimmte Berufsgruppen sowie die Personalverwaltung auf allen Verwaltungsebenen zuständig. Ihm ist das Prüfungsministerium und das Personalministerium unterstellt. Die hohe verfassungsrechtliche Bedeutung der Beamtenauslese geht auf die starke Stellung der Beamten im kaiserlichen China zurück.

Der Kontrollyuan ist das höchste Aufsichtsorgan der Regierung. Seine Kompetenzen umfassen die Beamten- und Ministeranklage, die Überprüfung der Amtsführung und die Rechnungskontrolle. $\mathrm{Ihm}$ ist das Ministerium für Rechnungsprüfung zugeordnet. Der Kontrollyuan setzt sich aus einem Präsidenten, einem Vizepräsidenten sowie 27 weiteren Mitgliedern zusammen, die vom Präsidenten für eine sechsjährige Amtszeit ernannt und von der Nationalversammlung bestätigt werden müssen.

Die übrigen Mitglieder des Exekutivyuan (Minister und Vorsitzende von Kommissionen und Ausschüssen) werden vom Präsidenten auf Vorschlag des Premierministers ernannt.

Schneider in Schubert/Schneider, Taiwan an der Schwelle zum 21. Jahrhundert(1996), S. 14.

60

Es fehlt eine genaue Kompetenzaufteilung zwischen den einzelnen Regierungsgewalten. Insbesondere die beiden in westlichen Verfassungssystemen unbekannten Gewalten des Kontrollyuan und des Prüfungsyuan funktionierten nie im Sinne Sun Yatsens. Der Kontrollyuan hatte nicht genug Macht, um die erhebliche Korruption und Misswirtschaft zu verhindern oder öffentlich bekannt zu machen, der Prüfungsyuan konnte die 1905 abgeschafften konfuzianisch geprägten Beamtenstaatsprüpfungen nicht ersetzen und ist nach Einführung geregelter Studiengängen obsolet 
fassungssystem als ein Mischsystem aus präsidialen und parlamentarischen Elementen zu bezeichnen.

\section{Die Verfassung und Verfassungspraxis von 1947 bis 1989}

Nach 1948 geriet die Regierung unter Chiang Kaishek immer mehr unter Druck: Zum einen musste sie sich im Bürgerkrieg der Großoffensive der immer stärker werdenden Roten Armee, zum anderen der Aufstände der Bevölkerung erwehren ${ }^{61}$. Zur Bewältigung dieser Krise und zur Erweiterung ihrer Befugnisse erließ die Nationalversammlung am 18. April 1948 die "Provisorischen Artikel während der Periode der Mobilmachung zur Niederschlagung der Rebellion" (Prov. Art.) ${ }^{62}$. Formell änderten die ProvArt. mit Wirkung vom 23.

geworden. Zu dieser kritischen Betrachtung des Verfassungskonzepts, Heuser, JöR n.F. Vol. 41 (1993), S. 659, 662f.

61

Auch auf dem vom Bürgerkrieg verschonten Taiwan kam die GMD-Regierung unter Druck. Zum einen wurde das relativ wohlhabende Taiwan von den - von acht Jahren Krieg ausgezehrten Soldaten der Nationalregierung geplündert, zum anderen entfaltete Korruption und Monopolisierung der führenden Positionen in Wirtschaft und Verwaltung durch GMD-Parteimitglieder rasch negative Auswirkungen auf Taiwan. Bei einem Zwischenfall am 27. Februar 1947 gerieten Beamte des Monopolamtes mit einer Straßenhändlerin in einen handgreiflichen Konflikt, der viele Schaulustige anzog. Als die Frau verletzt wurde, griff die empörte Menge die Beamten an und verfolgte diese. Als einer der Beamten in Panik in die Menge schoss, und eine Person starb, kam es in Taipei und später in weiteren Städten Taiwans zur sogenannten "Großen Revolution vom 28. Februar", welche die Nationalregierung und der korrupte Gouverneur der Provinz Taiwans Chen Yi nur mit Truppenverstärkung vom Festland und einem Massaker an der taiwanesischen Zivilbevölkerung niedergeschlagen konnten. Zu den Ereignissen vom 28. Februar 1947 siehe: Weggel, a.a.O., S. 93ff.; Lasars, JöR n.F. Vol. 41 (1993), S. 597, 622f.; China aktuell 3/1998, S. 276; Chen/Reisman, Yale Law Journal Vol. 81 (1972), S. 599, 612. Die neuere Forschung geht von 10.000 Menschen aus, die während und unmittelbar nach der Niederschlagung der Revolte zu Tode kamen, Tsehan/Myers/Wou, A Tragic Beginning: The Taiwan Uprising of February 28, 1947, S. 155-164.

Eine deutsche Übersetzung findet sich bei Lasars, VRÜ Vol. 25 (1992), S. 154ff.

Art. 1: Der Staatspräsident kann während der Periode der Mobilmachung, zur Niederschlagung der Rebellion zur Vermeidung einer dringenden Gefahr für den Staat und das Volk oder zur Bekämpfung einer sehr schweren Finanz- oder Wirtschafskatastrophe mit Zustimmung des Exekutivyuan Notstandsmaßnahmen ergreifen, ohne an das in den Art. 39 oder 43 Verfassung bestimmte Verfahren gebunden zu sein.

Art. 2 Die oben erwähnten Notstandsmaßnahmen können vom Legislativyuan in dem Verfahren nach Art. 57 Ziffer 2 der Verfassung geändert oder aufgehoben werden.

Art. 3: Die Beendigung der Periode der Mobilmachung zur Niederschlagung der Rebellion wird vom Staatspräsidenten proklamiert oder auf Ersuchen des Legislativyuans vom Staatspräsidenten proklamiert.

Art. 4: Die Aufhebung oder Weitergeltung der Provisorischen Artikel muss von der Nationalversammlung bestimmt werden 
Mai 1948 die Verfassung nicht, verdrängten sie aber in Teilen ${ }^{63}$ und bedeuteten so eine Verfassungsdurchbrechung, was für zulässig galt ${ }^{64}$.

\section{a. Die Verfassungsänderungen durch die Provisorischen Artikel 1948 - 1989}

Die Verfassung von 1947 ist wegen ihres hohen politischen Symbolwertes und der Gültigkeit für Gesamtchina formell auch in der Folgezeit nicht geändert worden. Stattdessen hat das Verfassungsrecht materiell wiederholt über die Prov. Art. Änderungen erfahren. Das Recht zur präsidialer Notverordnungen blieb bis 1991 bestehen ${ }^{65}$.

Das Recht des Präsidenten, gemäß Art. 1 Prov. Art. (und entgegen Art. 39 der Verfassung) ohne Mitwirkung der Legislative Notmaßnahmen zu erlassen, wurde in der Verfassungspraxis Taiwans bis zum Ende der Gültigkeit der Prov. Art. 1991 Grundlage des stark genutzten präsidialen Notverordnungsrechts. Mittels dieses präsidialen Notverordnungsrechts verhängte Präsident Chiang Kaishek 1949 den Ausnahmezustand für ganz China ${ }^{66}$, wonach alle Freiheitsrechte des Einzelnen von der obersten zuständigen Militärbehörde beschränkt werden konnten und auch tatsächlich wurden ${ }^{67}$.

1960 novellierte man die Prov. Art.: Abweichend von Art. 47 der Verfassung war künftig nicht nur die einmalige, sondern auch die mehrfache Wiederwahl des Staatspräsidenten zulässig $^{68}$. Dadurch konnte Chiang Kaishek bis zu seinem Tod am 5. April 1975 Präsident bleiben. Ferner lag es allein in der Macht des Präsidenten, über das Ende der "Zeit der Unterdrückung der (kommunistischen) Rebellion" und damit der Prov. Art. zu bestimmen. Des Weiteren durfte fortan der Präsident die Nationalversammlung einberufen, so dass auch außerhalb ihrer normalen Sitzungsperioden jederzeit die Prov. Art. geändert werden

Siehe hierzu Lasars, VRÜ Vol. 25 (1992), S. 115,119; Weng, JöR n.F. Vol. 27 (1978), S. 535, 537; Bünger, ZaöRV Vol. 6 (1936), S. 286-302.

Die chinesischen Verfassung von 1947 orientierte sich u.a. auch an der Weimarer Verfassung und sah daher wie diese keine Sperre gegen eine solche Verfassungsänderung außerhalb der Verfassung vor, Weng, JöR n.F. Vol. 27 (1978), S. 535, 536; Lasars, VRÜ Vol. 25 (1992), S. 115, 126. In der nationalchinesischen Verfassungslehre ist die Zulässigkeit einer solchen Verfassungsdurchbrechung daher auch unumstritten, Yao, JöR n.F. Vol. 35 (1986), S. 618, 629; Lasars, VRÜ Vol. 25 (1992), S. 115, 122. In der Bundesrepublik Deutschland ist eine Verfassungsdurchbrechung gemäß Art. 79 I GG unzulässig. Yao, JöR n.F. Vol. 35 (1986), S. 618, 621.

Heuser, JöR n.F. Vol. 41 (1993), S. 659, 664.

67

Yao, JöR n.F. Vol. 35 (1986), S. 618, 623.

68

Änderung vom 11. März 1960. Siehe hierzu Weng, JöR n.F. Vol. 27 (1978), S. 535, 543. 
konnten $^{69}$. So konnte Chiang Kaishek am 1966 die Nationalversammlung zu einer außerordentlichen Sitzung einberufen. Diese beschloss eine (zweite) Änderung der Prov. Art. , wonach die Nationalversammlung weitere Gesetzgebungsbefugnisse erhielt ${ }^{70}$. Im selben Jahr wurde durch eine weitere (die dritte) Änderung der Staatspräsident ermächtigt, ein besonderes Organ zur Bestimmung der politischen Leitlinien zu schaffen ${ }^{71}$. Auf dieser rechtlichen Grundlage erließ Präsident Chiang Kaishek 1967 die Verordnung über die Errichtung des "Nationalen Sicherheitsrats" ${ }^{72}$. Dessen Mitglieder wurden vom Präsidenten ernannt, dem sie auch unterstanden. Der Nationale Sicherheitsrat wurde das de facto höchste Kommandoorgan für die Innen- und Verteidigungspolitik ${ }^{73}$, mittels dessen sich Präsident Chiang Kaishek eine von der Verfassung nicht vorgesehene Eingriffsmöglichkeit in die Regierungspolitik verschaffte, die keinerlei Kontrolle unterlag. Die schrittweise Umgestaltung $^{74}$ der Verfassungswirklichkeit durch die Prov. Art. stärkte die Stellung des Staatspräsidenten, der mit umfangreichen Vollmachten ausgestattet wurde ${ }^{75}$. Die Prov. Art. und die zahlreiche Notverordnungen des Präsidenten haben die rechtliche Grundlage für das repressives Regierungssystem Chiang Kaisheks und dessen weitgespannten Sicherheitsapparat geschaffen.

Art. 1 und 2 Prov. Art. blieben unverändert. Der neue Art. 3 Prov. Art. bestimmte die Zulässigkeit der mehrfachen Wiederwahl des Staatspräsidenten. Der neue Art. 5 Prov. Art. bestimmte, dass der Staatspräsident in seiner dritten Amtsperiode in angemessener Zeit eine (weitere) außerordentliche Tagung der Nationalversammlung einberufen konnte. Der neue Art. 6 Prov. Art. hob das in Art. 3 a.F. Prov. Art. bestimmte Recht des Legislativyuans, das Ende der "Zeit der Unterdrückung der (kommunistischen) Rebellion" und damit der Prov. Art. zu beantragen, auf, und beließ die Entscheidung über das Ende somit ausschließlich dem Präsidenten, der hierdurch weiter gestärkt wurde. Zu den Änderungen insgesamt siehe Weng, JöR n.F. Vol. 27 (1978), S. 535, 540f.

Änderung vom 11. März 1966.

Änderung vom 22. März 1966.

Erlass vom 1. Februar 1967. Siehe hierzu Yao, JöR n.F. Vol. 35 (1986), S. 618, 621.

Weng, JöR n.F. Vol. 27 (1978), S. 535, 546.

Zur Entwicklung des Verfassungsrechtes bis 1978, Weng JöR n.F. Vol. 27 (1978), S. 535ff.

In der Fassung vom 18. März 1972 hatte der Präsident gemäß Art. 1 ein Notverordnungsrecht. Das Notverordnungsrecht des Präsidenten war materiell betrachtet eine unbeschränkte Vollmacht zur Verfassungsänderung gewesen, da die Notverordnungen höherrangiges Recht waren und die Verfassung und alle bestehenden Gesetze aufheben oder ändern konnten, Yao JöR n.F. Vol. 35 (1986), S. 618, 627. Der Präsident hatte ferner gemäß Art. 4 das Recht zur Schaffung neuer Organe, gemäß Art. 6 das Recht zum Erlass von Verordnungen bzgl. der Regelung der Wahl der Vertreter der Volksorgane und gemäß Art. 10 das alleinige Recht zur Beendigung der Zeit der Mobilmachung und damit der Geltung der Prov. Art. Da die Mandatszeit der zentralen Vertretungsorgane keiner zeitlichen Beschränkung unterlag, solange die Periode der Mobilmachung zur Niederschlagung der Rebellion währte und nur der Präsident über ihre Fortdauer entschied, waren die Abgeordneten faktisch vom Staatspräsidenten abhängig. Dieser war zudem nicht mehr auf zwei Amtsperioden beschränkt, sondern konnte gemäß Art. 3 viermal wiedergewählt werden, sodass Chiang Kaishek bis zu seinem Tod am 5. April 1975 Staatspräsident war, Weng, JöR n.F. Vol. 27 (1978), S. 535, 543. 
Die Verfassungsdurchbrechung durch die Prov. Art. war zwar nach h.M. verfassungsrechtlich gedeckt. Die Rechtsqualität der Prov. Art. hingegen wird unterschiedlich beurteilt ${ }^{76}$. Weil die Prov. Art. von der verfassungsgebenden Nationalversammlung stammten und an das Ende der Verfassung angehängt sind, seien sie ein Teil von ihr. Die Mehrheit der Verfassungsexperten lehnt das ab mit der Begründung, die Prov. Art. hätten die Verfassung abgeändert und könnten somit als höherrangiges Recht materiell nicht deren Teil sein; sie entsprächen auch formell - aufgrund ihrer zeitlichen Befristung - nicht dem Anspruch nach uneingeschränkter Geltung des Verfassungsrechts ${ }^{77}$. Einer weiteren Ansicht zufolge sind die Prov. Art. eine Kriegsverfassung, da sie während der Geltung des Kriegsrechtes die Friedensverfassung außer Kraft gesetzt hätten. Hiergegen wird angeführt, dass die Prov. Art. mit anfänglich 4 und später 12 Artikeln gar keine komplexe, das Staatswesen regelnde Gesetzeseinheit gebildet hätten ${ }^{78}$. Einer dritten Ansicht zufolge waren die Prov. Art. eine Art Sonderverfassung, die als lex specialis zu der an sich gleichrangigen Verfassung galten. Rechtssystematisch wird hiergegen angeführt, nach dem formalen Stufenaufbau der innerstaatlichen Rechtsordnung müsse die Verfassung Vorrang vor allen anderen Rechtsnormen haben; eine lex specialis bilde dagegen in der Regel ein Sondergesetz mit Unterfällen zu einem Normalgesetz. Die Prov. Art. hingegen würden vielmehr "Gegenfälle" zur Verfassung bilden. Die vorherrschende Rechtslehre betrachtet die Prov. Art. als eine Art Ermächtigungsgesetz der verfassungsgebenden Nationalversammlung, da die Prov. Art. hauptsächlich die Stellung des Präsidenten, z.B. durch das präsidiale Notverordnungsrecht, gestärkt hätten ${ }^{79}$. Die h.L. betrachtet daher auch die zahlreichen Notverordnungen des Präsidenten als verfassungskonform. Die Prov. Art. und die auf ihrer Grundlage ergangenen Verordnungen des Präsidenten haben die Entwicklung eines Rechtsstaates verhindert ${ }^{80}$. Das Kriegsrecht und zahlreiche Notverordnungen schränkten die Grundrechte weitgehend ein.

\section{b. Die Verfassungspraxis hinsichtlich der Mitglieder der Verfassungsorgane}

Die Verfassungspraxis nach 1949 war maßgeblich dadurch geprägt, dass viele der Abgeordneten aufgrund des Bürgerkriegs nicht nach Taiwan gelangen konnten, verstarben oder krankheitsbedingt nicht mehr an Sitzungen teilnehmen konnten. In den Verfassungsorganen fehlten daher viele Mitglieder und die gesetzlichen Mindestquoren für die Beschlussfähigkeit der Verfassungsorgane wurde (teilweise) nicht mehr erreicht. Um die BeschlussfähigYao, JöR n.F. Vol. 35 (1986), S. 618, 632. 
keit zu erhalten, wurden daher die gesetzlichen Quoren zum Teil auf ein Fünftel gesenkt ${ }^{81}$. Für die Verfassungspraxis war jedoch wichtiger, dass mit dem Ende des Bürgerkrieges 1949 und der Konsolidierung der Macht der kommunistischen Regierung auf dem chinesischen Festland die nach Taipei geflohene Regierung in einem großen Teil des Staatsgebietes keine verfassungsgemäßen Wahlen mehr durchführen konnte. Neuwahlen hätten von der Nationalregierung daher nur auf Taiwan, also 1\% der verfassungsrechtlichen Staatsfläche durchgeführt werden können. Anfang der fünfziger Jahre endeten verfassungsrechtlich jedoch die Mandate der Abgeordneten: 1951 zunächst die der auf drei Jahre gewählten Mitglieder des Legislativyuan, 1954 dann die der auf sechs Jahre gewählten Mitglieder der Nationalversammlung und des Kontrollyuan.

Da Wahlen zum Legislativyuan von 1951 bis 1953 nicht durchführbar waren, blieben die Mitglieder des Legislativyuans auf Aufforderung des Präsidenten und mit Zustimmung des Exekutivyuan - ohne Rechtsgrundlage - jeweils ein weiteres Jahr im Amt. Gleiches galt nach Ablauf ihres Mandats für die Mitglieder des Kontrollyuans im Jahre 1954. Beide Organe verloren hierdurch ihre verfassungsrechtliche und politische Legitimität. Um verfassungsrechtliche Zweifel, zu beseitigen wurde eine verfassungsrechtliche Entscheidung der Versammlung der Hohen Richter des Justizyuan beantragt ${ }^{82}$. Diese war jedoch nach der Flucht der Nationalregierung nach Taiwan selbst personell stark vermindert und nicht mehr beschlussfähig. Präsident Chiang Kaishek ernannte daher - mit Zustimmung des Prüfungsyuans - zunächst sieben neue Hohe Richter ${ }^{83}$. Die solchermaßen wieder beschlussfähige Versammlung der Hohen Richter erklärte 1954 dann in der „Interpretation Nr. 31" die Fortsetzung der Mandate der Abgeordneten des Legislativyuans und Kontrollyuans für verfassungsgemä $\beta^{84}$ und ermöglichte somit für Jahrzehnte eine

Am 27. Dezember 1952 wurde die gesetzliche Mindestanwesenheit für die Beschlußfähigkeit im Legislativyuan auf ein Fünftel herabgesetzt.

Gemäß Art. 78 der Verfassung ist die Auslegung der Verfassung dem Justizyuan anvertraut, der nach Art. 79 zur Erfüllung dieser Aufgabe die Versammlung der Hohen Richter einrichtet. Zur Versammlung der Hohen Richter siehe Weng, JöR n.F. Vol. 27 (1978), S. 535, 551. Zu den Zuständigkeiten und zum Justizyuan siehe Weng, JöR n.F. Vol. 21 (1972), S. 639-662. Die ersten Mitglieder der Versammlung der Hohen Richter wurden am 15. Juli 1948 vom Staatspräsidenten mit Zustimmung des Prüfungsyuans ernannt. Nach ihrer ersten ordentlichen Sitzung am 15. September 1948 tagte sie bis zum 19. November 1948 insgesamt zwölf Mal und beschloss zwei Auslegungen. Wegen des Bürgerkrieges waren danach lange keine Sitzung mehr möglich, Weng, JöR n.F. Vol. 27 (1978), S. 535, 552.

Dies erfolgte am 15. März 1952, siehe hierzu Weng, JöR n.F. Vol. 27 (1978), S. 535, 552.

Begründet wurde dies mit der Undurchführbarkeit von Wahlen und der Tatsache, dass nur so ein verfassungsrechtlich verbotener Stillstand der zentralen Vertretungsorgane verhindert werden könne. Ein Auszug aus der Interpretation Nr. 31 der Versammlung der Hohen Richter findet sich auf Deutsch bei Lasars, VRÜ Vol. 25 (1992), S. 115,156. Siehe hierzu auch Weng, JöR n.F. Vol. 27 (1978), S. 535, 553. 
undemokratische Vertretungspraxis in den Verfassungsorganen ${ }^{85}$. 1972 wurde dann bestimmt, dass die Mandatszeit der zentralen Vertretungsorgane keinerlei zeitlicher Beschränkung unterlag, solange die Periode der Mobilmachung zur Niederschlagung der Rebellion andauerte ${ }^{86}$.

Hinsichtlich der ebenfalls 1954 auslaufenden Mandate der Mitglieder der Nationalversammlung berief sich die Staatsspitze auf die Verfahrensvorschrift des Art. 28 Abs. 2 der Verfassung, wonach das Mandat der Abgeordneten der Nationalversammlung erst endet, wenn die nächste Nationalversammlung zusammentritt. Da Neuwahlen in Gesamtchina unmöglich waren und folglich eine neue (zweite) Nationalversammlung nicht zusammentreten konnte, wurde das Mandat der ersten Nationalversammlung bis zum Zusammentreten einer zweiten, in Gesamtchina gewählten Nationalversammlung „gestreckt“. Es ist offenkundig, dass dies verfassungswidrig und undemokratisch war.

In der Nationalversammlung wurde 1959 nicht mehr die verfahrensrechtlich notwendige Zweidrittelmehrheit der gesetzlichen Mitglieder ${ }^{87}$ für eine weitere Verfassungsänderung oder einen Beschluss über die Fortgeltung ${ }^{88}$ der Prov. Art. erreicht, da von den 3045 gesetzlichen Abgeordneten der Nationalversammlung im Jahre 1959 nur noch 1511 amtierten $^{89}$. Die Versammlung der Hohen Richter setzte sich auch hier über verfassungsrechtliche Bedenken hinweg. Nach ihrer „Interpretation Nr. 85“ berechnet sich die gesetzliche Mitgliederzahl danach, wie viele der gesetzmäßig gewählten Mitglieder in der gegen-

Zu den rechtlichen Details dieser Auslegung, Lasars, JöR n.F. Vol. 41 (1993), S. 597, 625ff.

Bestimmung vom 29. Juni 1972, siehe hierzu Weng, JöR n.F. Vol. 27 (1978), S. 535, 541f.

87

Die Anzahl der gesetzlichen Mitglieder der Nationalversammlung war streitig, da gemäß $\S 4$ des Gesetzes über die Wahl der Mitglieder der Nationalversammlung von 19483045 Mitglieder der Nationalversammlung hätten gewählt werden sollen, infolge des Bürgerkriegs tatsächlich jedoch nur 2961 Mitglieder gewählt wurden und auf der konstituierenden Sitzung der Nationalversammlung nur 2841 gewählte Mitglieder erschienen. Die Zweidrittelmehrheit betrug nach Wahlgesetz also 2030 Abgeordnete und nach tatsächlich Gewählten 1974. Auf der zweiten Tagung der Nationalversammlung auf Taiwan im Jahre 1954 waren nur noch 1578 der gewählten Abgeordneten anwesend. Im Laufe der Jahre fielen weitere 95 Mitglieder aus, von denen 28 gemäß den Bestimmungen der Prov. Art. durch ehemalige Mitkandidaten ersetzt wurden, Weng, JöR n.F. Vol. 27 (1978), S. 535, 557.

Spätestens bis zum 25.12.1950 hätte die Nationalversammlung entscheiden müssen, ob die Prov. Art. in Kraft bleiben oder aufgehoben werden sollen, Weng, JöR n.F. Vol. 27 (1978), S. 535, 538; Yao, JöR n.F. Vol. 35 (1986), S. 618, 621. Erst am 17. März 1954 beschloss die Nationalversammlung jedoch, die Fortgeltung der Prov. Art. Verfassungsrechtlich war dies umstritten, da auch die Fortgeltung der Verfassungsänderung durch die Prov. Art. eine Verfassungsänderung darstellt und gemäß Art. 174 der Verfassung dies einer Zweidrittelmehrheit der gesetzlichen Mitglieder der Nationalversammlung bedurf hätte. Eine solche Mehrheit der Mitglieder der Nationalversammlung ist der Regierung jedoch nicht nach Taiwan gefolgt, Weng, JöR n.F. Vol. 27 (1978), S. 535,538 . 
wärtigen Situation in der Lage seien, auf den Tagungen der Nationalversammlung zu erscheinen ${ }^{90}$. Mit dieser rechtlich unvertretbaren Auslegung der Verfassung sicherte die Versammlung der Hohen Richter dauerhaft die Beschlussfähigkeit der Nationalversammlung.

Auf Grundlage der Art. 5 Prov. Art. wurde der Präsident ermächtigt, Regelungen über die Vertreter in der Nationalversammlung und in dem Legislativyuan zu erlassen ${ }^{91}$. Der Präsident erließ zunächst Vorschriften, welche es ermöglichten, dass bei schwerer Krankheit oder Tod eines Abgeordneten, dieser durch einen ehemaligen Mitkandidaten bei der Wahl von 1948 ersetzt werden konnte. Des Weiteren bestimmte er, dass für die Nationalversammlung 15, für den Legislativyuan elf und für den Kontrollyuan zwei neue Mitglieder gewählt werden. Dies wurde möglich, indem die Anzahl der aus Taiwan zu wählenden Abgeordneten erhöht und für sog. Auslandschinesen neue Abgeordnete eingeführt wurden - alles entgegen den Bestimmungen der Verfassung ${ }^{92}$. Diese Art der Zusatzwahlen fand fortan regelmäßig statt, wodurch das Durchschnittsalter von über 80 Jahren etwas gesenkt wurde.

Die Nationalversammlung mit einer gesetzlichen Sollstärke von über 3045 Abgeordneten bestand im Januar 1989 nur noch aus 757 Abgeordneten, davon 673 sogenannte "ewigen" Abgeordneten, die sich seit mehr als vier Jahrzehnten keiner Wahl mehr stellen mussten ${ }^{93}$.

\begin{tabular}{|l|c|c|c|c|}
\hline & Kontroll-Yuan & Legislativ-Yuan & $\begin{array}{c}\text { National- } \\
\text { versammlung }\end{array}$ & Total \\
\hline Gesetzliche Mitglieder & 223 & 773 & 3045 & 4041 \\
\hline $\begin{array}{l}\text { Gewählte Mitglieder } \\
(1947 / 1948)\end{array}$ & 180 & 757 & 2953 & 3890 \\
\hline Abgeordnete aus Taiwan & 5 & 8 & 19 & 32 \\
\hline
\end{tabular}

„Interpretation Nr. 85“ vom 12. Februar 1960, siehe hierzu Weng, JöR n.F. Vol. 27 (1978), S. $535,557$.

Art. 5: "To meet the requirements of national mobilisation and the suppression of rebellion, the President may make adjustments in the administrative and personal organs of the central government as well as their organization." Siehe hierzu Weng, JöR n.F. Vol. 27 (1978), S. 535, 540f..

Die sogenannten Auslandschinesen konnten 15 Mitglieder des Legislativyuans und fünf des Prüfungsyuans wählen.

Bei der Wahl des Präsidenten 1990 wurden 21 unheilbar kranke beziehungsweise vom Alter geschwächte Abgeordnete, die kaum mehr zu eigenen Bewegungen fähig waren, von Familienmitgliedern und Krankenschwestern in die Nationalversammlung begleitet, Lasars, VRÜ Vol. 25 (1992), S. 115, 121. 


\begin{tabular}{|l|c|c|c|c|}
\hline $\begin{array}{l}1949 \text { nach Taiwan gelangte } \\
\text { Abgeordnete }\end{array}$ & 102 & 551 & 1643 & 2296 \\
\hline $\begin{array}{l}\text { "Ewige" Abgeordnete } \\
(3 / 1990)\end{array}$ & 22 & 152 & 673 & 847 \\
\hline Neue Abgeordnete (3/1990) & 31 & 127 & 84 & 242 \\
\hline
\end{tabular}

Verfassungsrechtlich fehlte den Volksvertretern somit sowohl für Taiwan als auch für das Festland eine demokratische Vertretungsbefugnis ${ }^{94}$. Die ewigen Abgeordneten repräsentierten die verschiedenen Provinzen Festlandchinas und fungierten nach der Flucht der Regierung um Chiang Kaishek als Symbol für den Alleinvertretungsanspruchs der Regierung Taipehs ${ }^{95}$.

\section{c. Zwischenergebnis}

Trotz der Teilung Chinas seit 1949 wurde von der Regierung der Republik China in der Verfassungspraxis bis 1989 durch die Prov. Artikel und die Perpetuierung der Mandate der gewählten Volksvertreter der unveränderte Fortbestand der Republik China propagiert und verfassungsrechtlich exerziert. Es wurde systematisch versucht, verfassungsrechtlich die politische Illusion einer unverändert fortbestehenden Republik China in den Grenzen von 1942 zu kreieren. Dies diente innenpolitisch der Pseudolegitimierung der GMD-Regierung, und gegenüber der Volksrepublik China zur Aufrechterhaltung der Ein-China-Politik.

\section{Die Verfassung und Verfassungspraxis nach 1989}

Die Verfassungspraxis im Herrschaftsgebiet der Regierung der Republik China ist nach 1989 entscheidend von der Demokratisierung und der hiermit in Verbindung stehenden Einführung ergänzender Verfassungszusätze geprägt worden ${ }^{96}$. Noch unter der Präsidentschaft Jiang Jingguos, wurde am 14. Juli 1987 nach 38 Jahren, nicht zuletzt aufgrund stetig 25 (1992), S. 115, 122ff.; Lasars, JöR n.F. Vol. 41 (1993), S. 597, $625 f f$. Schubert, a.a.O., S. 17, 19.

96 Zur Bedeutung der Demokratisierung Taiwans in den innerchinesischen Beziehungen siehe: Dickson, Asian Survey Vol. 38 (1998) Nr. 4, S. 349-364; Jia, Journal of Northeast Asian Studies Vol. 13 (1994) Nr. 1, S. 49-63; Kau, in Wheeler, Chinese divide. Evolving relations between Taiwan and Mainland China (1996), S. 47-71; Moody, Journal of East Asian Affairs Vol. 5 (1991) Nr. 1, S. 144-184. 
steigender in- und ausländischer Proteste ${ }^{97}$, das Kriegsrecht aufgehoben. Mit dem Tode Jiang Jingguos 1988 endete das autoritäre Herrschaftssystem der GMD, und Taiwan begann, sich zu demokratisieren ${ }^{98}$ : Politische Parteien wurden zugelassen ${ }^{99}$, die ZwölfSeiten-Limitierung ${ }^{100}$ der Zeitungen ebenso wie die Pressezensur aufgehoben, neue (unabhängige) Zeitungen zugelassen, und Demonstrationen als Form politischer Beteiligung erlaubt.

Die Demokratisierung verlief verfassungsrechtlich in mehreren Schritten. Zunächst wurde den ewigen Abgeordneten durch das am 3. Februar 1989 verabschiedete "RücktrittsGesetz" 101 ein Rücktrittsrecht angeboten. Abgeordneten, die ihr Mandat seit mehr als einem Jahr nicht mehr ausübten, sei es aus gesundheitlichen Gründen oder weil sie im Ausland lebten, wurde ein freiwilliger Rücktritt unterstellt ${ }^{102}$. Da die meisten der ewigen Abgeordneten jedoch weiterhin an ihrem Mandat festhielten, verfehlte dieses Gesetz die beabsichtigte Wirkung. Daraufhin wurde die Versammlung der Hohen Richter angerufen. Diese entschied am 21. Juni 1990 in der sensationellen „Interpretation Nr. 261“103, dass alle in den Jahren 1947/48 noch auf dem chinesischen Festland gewählten ewigen Abgeordneten der drei zentralen Volksvertretungsorgane zum 31. Dezember 1991 pensioniert werden müssten und Neuwahlen für die zentralen Volksvertretungsorgane durchzuführen seien $^{104}$. Durch diese Entscheidung wurden die ersten demokratischen Wahlen auf Taiwan

Organisierte politische Oppositionsgruppen und soziale Bewegungen forderten schon zu Beginn der Achtziger immer vehementer eine Liberalisierung des politischen Systems, Chen, Diss. München 1992, $196 f$.

Lasars, VRÜ Vol. 25 (1992), S. 115-160; Heuser, JöR n.F. Vol. 41 (1993), S. 659-671.

Zur Demokratisierung in Taiwan siehe auch: Domes, Asian Survey Vol. 21 (1981) Nr. 10, S. 1011-1028; Chou/Nathan, Asian Survey Vol. 27 (1987) Nr. 3, S. 277-299; Lee, Politique Internationale Vol. 72 (1996), S. 5-13; Klintworth, New Taiwan, New China (1995), S. 231ff.

100

Diese sogenannte Maulkorbbestimmung hatte über Jahrzehnte die Entwicklung einer kritischen und ausführlichen Berichterstattung in den Zeitungen verhindert.

Das "Gesetz über den freiwilligen Rücktritt der alten und erfahrenen Volksvertreter der Ersten Zentralen Vertretungsorgane".

102

103

Lasars, VRÜ, Vol. 25 (1992), S. 115, 122.

Ein auszugsweiser Abdruck der Interpretation Nr. 261 findet sich auf Deutsch bei Lasars, VRÜ Vol. 25 (1992), S. 115, 157.

104

Die Interpretation wurde von den ewigen Abgeordneten stark kritisiert und als politisches Urteil bezeichnet. Tatsächlich enthält die Verfassung, über deren Auslegung die Richter ausschließlich zu befinden hatten, keine Bestimmungen, die eine Fristsetzung für den 31. Dezember 1991 vorsehen. Die Richter hielten sich mit ihrer Auslegung vielmehr genau an den von der GMD-Spitze und dem neuen Präsident Li mit den Oppositionsführern vereinbarten Zeitplan für Reformen. Es handelt sich, politisch betrachtet, um eine späte, aber notwendige Korrektur der Auslegung Nr. 31, welche die Mandate der Abgeordneten ohne Wahlen fortgelten ließ und dabei gegen die demokratischen Bestimmungen der Verfassung verstieß, Lasars, VRÜ Vol. 25 (1992), S. 115,122. 
ermöglicht, zugleich jedoch eine Verfassungsreform unumgänglich ${ }^{105}$, da Neuwahlen entgegen der Verfassung nicht in Gesamtchina durchgeführt werden konnten. Am 1. Mai 1991 wurde dann die "Mobilisierung für die Zeit der Niederschlagung der kommunistischen Rebellion" außer Kraft gesetzt. Dies wiederum ermöglichte die Aufhebung der Prov. $\mathrm{Art}^{106}$. Damit war der Weg frei für eine weitere Demokratisierung und Verfassungsreform.

Bei der Demokratisierung stellte sich die Frage, wie verfassungsrechtlich Wahlen ermöglicht werden konnten, ohne die Verfassung formal zu ändern und ohne verfassungsrechtlich die Einheit Chinas aufzugeben. Innerhalb der GMD herrschte Einigkeit, dass die Ausarbeitung und Verkündung einer neuen Verfassung nicht in Frage kam, da befürchtet wurde, die Volksrepublik könnte eine neue Verfassung als Schritt in die Unabhängigkeit interpretieren $^{107}$.

Um einerseits die Verfassung von $1947 \mathrm{zu}$ wahren und andererseits die Verfassung den demokratischen Bedürfnissen Taiwans anzupassen, ging man über zum Konzept der Verfassungszusätze nach dem amerikanischen Amendment-Prinzip.

\section{a. Die Verfassungsänderung von 1991}

Am 1. Mai 1991 wurde ein Verfassungszusatz (VerfZ) ${ }^{108}$ mit zehn Artikeln verkündet. Nach Art. 1 des VerfZ werden - abweichend von den Wahlbestimmungen in der Verfassung - für die Wahl der Nationalversammlung die Abgeordneten im Herrschaftsbereich der Regierung der Republik China direkt gewählt. Im übrigen wählen im Ausland ansässige Staatsbürger 20 Abgeordnete, und für das übrige Staatsgebiet werden von den Wählern im Herrschaftsbereich der Regierung der Republik China achtzig Abgeordnete für und in einem nationalen Wahlkreis gewählt ${ }^{109}$. Die Fiktion einer sich auf ganz China erstrecken-

105

106

Lasars, VRÜ Vol. 25 (1992), S. 115, 122.

Lasars, VRÜ Vol. 25 (1992), S. 115-160; Heuser, JöR n.F. Vol. 41 (1993), S. 659-671. Bereits im Juli 1990 hatte Präsident Li Denghui die "Konferenz über nationale Angelegenheiten" einberufen, an der Vertreter aus Regierung, Parteien, Wissenschaft, Wirtschaft und Gesellschaft teilnahmen, die sich - trotz unterschiedlicher politischer Auffassung - darauf einigten, dass zunächst die 107 Prov. Art. aufgehoben werden müssten.

$W u$, Streitbare Demokratie, S. 124.

108

Ein Abdruck des Verfassungszusatzes findet sich auf deutsch bei Lasars, VRÜ Vol. 25 (1992), S. 115, 157ff. und bei Heuser, JöR n.F. Vol. 41 (1993), 659, 669ff.

109

In jeder regierungsunmittelbaren Stadt, in jedem Kreis und in jeder Stadt werden je zwei Abgeordnete gewählt. Überschreitet deren Bevölkerung 100.000 Einwohner, wird für jeweils weitere 100.000 Einwohner ein weiterer Abgeordneter gewählt. Den "Ureinwohnern des Flachlandes und der Bergregion" Taiwans stehen zusätzlich drei Abgeordnete zu. 
den Volksvertretung ist jedoch nicht aufgegeben ${ }^{110}$, da 80 Abgeordnete der Nationalversammlung nicht für und in einem bestimmten Wahlkreis auf Taiwan, sondern von allen Bürgern im Herrschaftsbereich der Republik China für Gesamtchina gewählt wurden. Weitere Regelungen des VerfZ in diesem Zusammenhang müssen hier nicht dargestellt werden $^{111}$.

Art. 5 Abs. 1 VerfZ bestimmte, dass die Delegierten der Zweiten Nationalversammlung vor dem 31. Dezember 1991 gewählt sein, die Amtszeit am 1. Januar 1992 beginnen und im Jahre 1996 enden musste ${ }^{112}$. Entsprechende Regelungen wurden für den Legislativyuan und den Kontrollyuan erlassen ${ }^{113}$. Entsprechend der Regelung in VerfZ (1991) wurde am 21. Dezember 1991 - in den ersten wirklich freien und allgemeinen Wahlen auf Taiwan eine Nationalversammlung gewählt, die aus 325 Neumitgliedern und den nach den Prov. Art. neu gewählten Abgeordneten bestand, deren Mandat erst am 31.1.1993 endete. Bei den Wahlen zur Nationalversammlung 1991 konnten die GMD 254 (71\%), die Demokratische Fortschrittspartei (DFP) ${ }^{114} 66(23 \%)$ und andere Parteien $5(6 \%)$ Mandate erringen ${ }^{115}$.

110

Dies ergibt sich aus den Artikeln 1 Abs. 4 und 2 Abs. 4 VerfZ (1992), die die Wahl von 80 Mandaten in der Nationalversammlung beziehungsweise 30 im Legislativyuan als Abgeordnete Gesamtchinas ohne eigenen Wahlkreis vorschreiben.

111 Kritisiert wurde zudem, dass gemäß Art. 7 VerfZ (1991) der Präsident wieder ein Notstandsrecht erhielt (wenngleich der Legislativyuan nun zustimmen muss) und der Präsident gemäß Art. 9 VerfZ (1991) einen Nationalen Sicherheitsrat einrichten konnte, der ihm nachgeordnet ist.

Die zusätzlichen Abgeordneten, die gemäß den Prov. Art. gewählt wurden, sollten gemäß Art. 5 Abs. 2 VerfZ 1991 ihr Mandat zusammen mit den Abgeordneten der Zweiten Nationalversammlung bis zum 31.1.1993 ausüben.

113

Art. 5 Abs. 3 VerfZ bestimmte, dass die Abgeordneten des Zweiten Legislativyuan und des Zweiten Kontrollyuan bis zum 31. Januar 1993 gewählt werden sollten, und ihre Amtsperiode am 1. Februar 1993 beginnen müsse. In jeder Provinz und in Stadt im Herrschaftsbereich der Regierung der Republik China, werden je zwei Abgeordnete gewählt. Überschreitet deren Bevölkerung 200.000 Einwohner wird für jeweils weitere 100.000 Einwohner ein weiterer Abgeordneter gewählt. Übersteigt die Bevölkerung eine Million Einwohner, wird für jeweils weitere 200.000 Einwohner ein weiterer Abgeordneter gewählt. Den Ureinwohner des Flachlandes und der Bergregion stehen zusätzlich drei Abgeordnete zu. Ständig im Ausland ansässige Staatsbürger wählen sechs Abgeordnete und für das übrige Staatsgebiet werden dreißig Delegierte in einem nationalen Wahlkreis gewählt.

114 Zur Abkürzung: Gemäß der englischen Übersetzung des Parteinamens, "Democratic Progressive Party", findet sich mitunter auch in der deutschsprachigen Literatur und Presse das Kürzel "DPP" 115 sowie die chinesische Abkürzung "MCT" (Minzhu jinbudang).

Zu dieser Wahl siehe Schubert, Taiwan die chinesische Alternative (1994), S. 53. 


\section{b. Die Verfassungsänderung von 1992}

Gemäß Art. 6 des VerfZ berief Präsident Li Denghui ${ }^{116}$ nach der Wahl die Mitglieder der Zweiten Nationalversammlung ein, die auf einer außerordentlichen Sitzung über eine weitere Verfassungsreform beratschlagen sollte ${ }^{117}$. Innerhalb der GMD kam es zu verschiedenen Vorschlägen und einem erbitterten verfassungsrechtlichen Streit, in dessen Folge einige Abgeordnete der GMD sich abspalteten und die "Neue Partei Chinas" gründeten ${ }^{118}$. Der Entwurf der DFP für eine komplett neue Verfassung mit einem präsidialen DreiGewaltensystem wurde abgelehnt ${ }^{119}$ und am 27. Mai 1992 der Vorschlag der Mehrheit der GMD, den Verfassungszusatz um acht weitere Artikel zu ergänzen, angenommen ${ }^{120}$. Die ergänzenden Artikel in der Fassung von 1992 (VerfZ 1992) stärkten die Nationalversammlung und den Präsidenten auf Kosten des Legislativ-, Kontroll- und Exekutivyuan ${ }^{121}$. Dafür wurden die Amtszeit des Präsidenten und die Legislaturperiode der Nationalversammlung von sechs auf vier Jahre verkürzt. Darüber hinaus wurden die Kompetenzen und die Organisation von Justiz-, Prüfungs- und Kontrollyuan neu geregelt, und eine demokratische Kreisverwaltung eingeführt ${ }^{122}$.

Auch diese Verfassungsänderung hielt formell an der Verfassung von 1947 fest. Zum Beispiel ist die in Art. 6 erwähnte Flagge identisch mit der vorher für Gesamtchina geltenden Flagge. Die gesamte Verfassung geht zudem vom Fortbestand des zuvor auf dem chinesischen Festland existierenden einen Herrschaftsverbandes aus. Darauf deuten die zahlreichen Erwähnungen der unterschiedlichen Volksgruppen, der Autonomierechte (z.B. für Tibet), die Kompetenzverteilung zwischen Zentral- und Provinzregierung (Artt. 107ff) und die Aufteilung in Provinzen (Art. 112) hin. Die Verfassung von 1947 geht also auch nach den Verfassungszusatz von 2004 weiter fest vom Fortbestand des zur Zeit der Verfassungs-

Zu Li Denghui siehe Halbeisen, Eine Ära Lee Teng-hui?(1997).

118 Siehe hierzu Schneider in Schubert/Schneider, a.a.O., S. 25.

119

120 Der 2. Verfassungszusatz von 1992 findet sich auf Deutsch bei Heuser, JöR n.F. Vol. 41 (1993), $659,669 \mathrm{ff}$.

121

Der Präsident erhielt vorbehaltlich der Zustimmung der Nationalversammlung das Recht zur Ernennung der bisher direkt gewählten Mitglieder des Kontrollyuan (Art. 15 VerfZ). Der Kontrollyuan wurde durch den Entzug der direkten demokratischen Legitimierung zu einer Art Justizorgan umgeformt und verlor auch das Recht der Zustimmung bei Ernennung von Mitgliedern des Justiz- und Prüfungsyuans, welches ebenfalls der Nationalversammlung übertragen wurde (Art. 13, 14 VerfZ (1992)).

122 Art. 17 VerfZ 1992 sieht vor, dass jede Provinz einen Landtag und eine Provinzregierung mit Provinzgouverneur und jeder Kreis einen Kreistag mit Kreisregierung und einen Landrat an der Spitze wählt. 
gebung existenten chinesischen Staates mit all seinen Provinzen aus ${ }^{123}$. Aufgrund ihrer weitgehenden Änderungen durch die VerfZ wurde die Verfassung zu Recht als "eine vom tatsächlichen Leben getrennte Ideal- oder Illusionsverfassung" bezeichnet ${ }^{124}$. Diese Verfassungsfiktion zeigte sich besonders deutlich daran, dass nach dem VerfZ 1992 nunmehr fast alle Abgeordneten der Nationalversammlung im Herrschaftsbereich der Regierung der Republik China gewählt wurden, obwohl nach der Verfassung von 1947 nur zehn von 3045 Abgeordneten auf Taiwan gewählt werden sollten ${ }^{125}$

$c$.

\section{Die Verfassungsänderung von 1994}

Da Art. 12 des VerfZ 1992 eine Fortführung der Verfassungsreform ausdrücklich vorschrieb, bedurfte es einer weiteren Verfassungsergänzung ${ }^{126}$. Auf der 4. Plenarsitzung der Zweiten Nationalversammlung wurde am 29. Juli 1994 daher nach langer Erörterung und Diskussion die dritte Verfassungsergänzung beschlossen (VerfZ 1994) ${ }^{127}$. Diese reduzierte die 18 Zusatzartikel auf zehn. Inhaltlich wurde das Wahlrecht erneut geändert und die Nationalversammlung weiter gestärkt ${ }^{128}$. Außerdem wurde ein starkes demokratisches Element, die Direktwahl des Präsidenten, eingeführt. Am 23. März 1996 gab es die ersten direkten Präsidentschaftswahlen. Bei einer Wahlbeteiligung von $76 \%$ gewann der bisherige Präsident und GMD-Politiker Li Denghui mit $54 \%$ der Stimmen. Der Kandidat der DFP, Peng Mingmin, erhielt $21.1 \%$ der Stimmen und auf die unabhängigen Kandidaten Lin Yanggang und Chen Lüan entfielen $10 \%$ beziehungsweise $14.9 \%$ der Stimmen ${ }^{129}$. In dieser zweiten Stufe der Verfassungsreform wurde auch die Direktwahl des Provinzgouverneurs von Taiwan eingeführt, welche der GMD-Politiker James Soong gewann.

Das Regierungssystem dokumentierte auch nach 1994 formell weiterhin nicht den Willen, auf verfassungsrechtlicher Ebene als eigenständiger, von der Volksrepublik China unabhängiger Staat zu fungieren ${ }^{130}$, da die gesamte Verfassungsstruktur weiterhin von dem

Clough, CYILA Vol. 1 (1981), S. 17, $17 \mathrm{f}$.

Heuser, JöR n.F. Vol. 41 (1993), 659, 666.

125 Heuser, JöR n.F. Vol. 41 (1993), 659, 666.

126

Siehe hierzu Schneider in Schubert/Schneider, a.a.O., S. 25.

127 Dieser dritte Verfassungszusatz wurde gegen die Stimmen der DFP und Teile der GMD beschlossen und am 1. August 1994 von Präsident Li Denghui verkündet.

Die Nationalversammlung kann seither über ihre innere Organisation selbst bestimmen (Art. 1 Abs. 8) und im Falle eines Amtsenthebungsverfahrens gegen den Präsidenten durch ihren selbstgewählten Sprecher die Nationalversammlung unabhängig von der Zustimmung des Legislativyuan einberufen (Art. 1 Abs. 8)

130

Zu den Wahlergebnissen siehe Nieh, China aktuell,3/1996, S. 262-264.

Schubert, a.a.O., S. 17, 23.
} 
Fortbestand eines Staates China ausgeht und die VerfZ lediglich "provisorische" Regelungen wegen der Undurchführbarkeit freier Wahlen auf dem Festland aufstellen.

\section{d. Die Verfassungsänderung von 1997}

Die Probleme der Verfassungsstruktur von 1947 wurden durch die drei Stufen der Verfassungsreform und die Verfassungszusätze von 1991, 1992 und 1994 nicht nur nicht behoben, sondern zum Teil noch verschärft und machten eine vierte Phase der Verfassungsreform nötig ${ }^{131}$. Nach den Wahlen zur Dritten Nationalversammlung $1996^{132}$ wurde eine Verfassungsänderung jedoch erheblich schwerer, weil die GMD nicht mehr über eine verfassungsändernde Mehrheit verfügte, und somit parteiübergreifende Konsensvorschläge erarbeitet werden mussten ${ }^{133}$. Die hierbei geschlossenen politischen Kompromisse gingen zum Teil auf Kosten der Rechtssystematik.

1997 brachte die nächste Verfassungsergänzung (VerfZ 1997). Sie gestaltete das Verhältnis des Staatspräsidenten zur Regierung neu. Der Staatspräsident hat seither die Richtlinienkompetenz für die Innen- und Außenpolitik, was das Regierungssystem in Richtung Präsidialdemokratie verschiebt. Das Amt des Premierministers und der Exekutivyuan büßten folgerichtig an Bedeutung ein: Der Premier wird seither nunmehr allein vom Präsidenten ernannt, und seine Ernennung bedarf nicht mehr der Zustimmung des Legislativyuan ${ }^{134}$. Der Legislativyuan erhielt im Gegenzug das Recht, mit einem Misstrauensvotum den Premierminister zum Rücktritt zu zwingen. Außerdem wurden die Amtszeiten von Legislativund Exekutivyuan synchronisiert.

Schneider in Schubert/Schneider, a.a.O., S. 29. Durch den Machtkampf zwischen Legislativyuan und Präsident um die Ernennung des Premierministers kam es zu einer Blockade des politischen Systems, vgl. Tränkmann, Asien Nr. 67 (1998), S. 53, 53f.

Das Wahlrecht in Taiwan ist unüblich, womöglich einzigartig. Für die dritte Nationalversammlung 1996 wurden 334 Sitze vergeben, davon nach Mehrheitswahlrecht sechs für die Eingeborenen Taiwans, 228 für die 56 Distrikte und die übrigen 100 nach einem Verhältniswahlrecht mit Fünfprozenthürde, wovon 80 die nationale Wählerschaft und 20 die Auslandschinesen vertreten. Zu diesem Wahlrecht im Detail siehe Moon, Asian Survey Vol. 40 (2000) Nr. 1, S. 652-668.

Bei den Wahlen 1996 konnte die GMD 54,79\% der Stimmen (183 von 334 Sitzen), die DFP $29,64 \%$ (99 Sitze), Unabhängige 1,80\% (6 Sitze) und die Neue Partei 13,77\% (46 Sitze) erreichen. Dadurch verfügte keine Partei mehr über die notwendige Mehrheit für eine Verfassungsveränderung. Zu den Wahlergebnissen siehe Nieh, in China aktuell, 3/1996, S. 262-264.

Freies Asien Nr. 23, 19.12.1997, S. 3. 
Die Anzahl der Abgeordneten im Legislativyuan erhöhte man von 165 auf 225 Abgeordnete $^{135}$.

Die Verwaltung wurde durch eine Verringerung der Kompetenzen der Provinzregierung und Erweiterung der Befugnisse der Zentralregierung verschlankt ${ }^{136}$. Von einem hierarchischen dreigliedrigen Staatsaufbau hat sich Taiwan hierdurch verabschiedet und die Kompetenzen der zweiten Ebene, der Provinzregierung, überwiegend auf die Zentralregierung, aber auch zugunsten der kommunalen Selbstverwaltung, verlagert. Dennoch wurde grundsätzlich die schwerfällige Struktur mit einer Zentralregierung, der Regierung der Provinz Taiwan und der Regierung der Provinz Fujian sowie den Kreisregierungen aufrechterhalten. Die bisherige Einteilung in Provinzen wurde aufrechterhalten, obwohl der Herrschaftsbereich der Regierung der Republik China von den ursprünglichen 35 Provinzen vollständig nur die Provinz Taiwan und zwei Kreise der Provinz Fujian (Mazu und Jinmen) umfasst $^{137}$. Der Staatsaufbau wurde insgesamt also trotz langer Diskussionen ausdrücklich beibehalten, da dies den Anspruch aufrechterhält, die Regierung der schon 1911 existierenden Republik China zu sein. Es sollte bewusst der Eindruck vermieden werden, dass es sich in Taipei um eine neue Regierung eines unabhängigen Staates Taiwan handelt.

Das Regierungssystem dokumentierte daher auch nach 1997 formell weiterhin nicht den Willen, auf verfassungsrechtlicher Ebene als eigenständiger, von der Volksrepublik China unabhängiger Staat zu fungieren, da die gesamte Verfassungsstruktur weiterhin von dem Fortbestand eines Staates China ausgeht. Auch das Wahlrecht lässt weiter das Festhalten an der Einheit Chinas erkennen ${ }^{138}$. Hwang, Free China Review Vol. 12 (1999) Nr. 10, S. 21; Liao/Tsai, Issues and Studies Vol. 37 (2001) Nr. 3, S. 1-31; McBeath, Asian Survey Vol. 40 (2000) Nr. 2, 251-268.

136 Die erst durch die Verfassungsreform 1994 eingeführte Direktwahl des Gouverneurs wurde rückgängig gemacht. Seit der Verfassungsreform 1997 wird die Provinzregierung wieder vom Premierminister nominiert und vom Präsidenten ernannt und die Provinzversammlung zu einem beratenden Organ, deren Mitglieder durch das gleiche Verfahren ernannt werden.

Die Provinz Taiwan umfasst 98\% des Herrschaftsbereichs der Regierung der Republik China. Die Provinzregierung verlor im Dezember 1998 große Teile ihre Befugnisse und ist nunmehr weitgehend nur noch ein untergeordnetes Organ der Zentralregierung. Der Provinzgouverneur wird nicht 138 mehr gewählt, sondern von der Zentralregierung ernannt, China aktuell 12/1998, S. 1310.

Das Parlament, der Legislativyuan, hat 225 Sitze und jeder Bürger nur eine Stimme. Die Stimmberechtigten gliedern sich in die Gruppen der "normalen" Wähler, der Ureinwohner und der Auslandschinesen. Die Gruppe der "normalen" Wähler wählen in Wahlkreisen 168 Direktmandate. Während in Deutschland pro Wahlkreis lediglich ein Abgeordneter direkt gewählt wird, sind es in Taiwan je nach Größe des Wahlkreises jedoch zum Teil über zehn. Unabhängig von einer Mindeststimmenzahl zieht dann eine entsprechende Anzahl von Abgeordneten in das Parlament. Vereinigen drei Kandidaten in einem Wahlkreis mit vier Abgeordneten je $32 \%$ der Stimmen auf sich, erhält Kandidat 4 mit $4 \%$ der Stimmen ebenfalls ein Mandat. Daher haben Unabhängige besonders gute Chancen. Die Ureinwohner wählen in einem eigenen Wahlkreis acht Abgeordnete 


\section{e. $\quad$ Die Verfassungsänderung von 1999 und 2000}

Seit der Geltung des VerfZ 1997 war die Nationalversammlung nur noch für Verfassungsänderungen, die Wahl des Vizepräsidenten und einige andere (eher unbedeutende) Funktionen zuständig, die auch durch den Legislativyuan wahrgenommen werden könnten. Daher einigten sich die beiden größten Parteien, die GMD und die DFP, auf eine Verfassungsänderung zur Abschaffung der Nationalversammlung. In einem ersten Schritt hierzu sollten die Amtszeiten der beiden Parlamente, der Nationalversammlung und des Legislativyuans synchronisiert werden, da die Nationalversammlung im Mai 1996 auf vier Jahre und der Legislativyuan im Dezember 1998 auf drei Jahre gewählt wurden. Durch eine Verfassungsänderung beschloss die Nationalversammlung daher zum einen die Verlängerung ihrer eigenen Amtszeit, die bis zum 19. Mai 2000 gehen sollte, um zwei Jahre bis Ende Juni 2002, und zum anderen, die einmalige Verlängerung der Legislaturperiode des Legislativyuan um ein halbes Jahr bis ebenfalls Ende Juni 2002. Von da an sollte die Nationalversammlung nicht mehr vom Volk gewählt, sondern nach dem Parteienproporz des Legislativyuan entsprechend besetzt werden. Diese Verfassungsänderung wurde jedoch von der Versammlung der Hohen Richter am 24. März 2000 verworfen: Die eigene Amtszeit zu verlängern, sei ein grober Rechtsverstoß ${ }^{139}$. Folglich galt ab sofort die Verfassung wieder in Gestalt der Änderung vom 21. Juli 1997.

Die GMD, die DFP und die New Party einigten sich daraufhin, die Nationalversammlung sofort abzuschaffen. In Umsetzung des höchstrichterlichen Urteils hob die Nationalversammlung daher zunächst ihren Beschluss zur Verlängerung der eigenen Amtszeit auf und beschloss am 24. April 2000, dass die Nationalversammlung nicht mehr, wie bisher, regelmäßig gewählt, sondern nur noch im Bedarfsfall und als ad-hoc Gremium vom Legislativyuan einberufen wird.

\section{f. Die Verfassungsänderung von 2004}

Der Legislativyuan hat im August 2004 dann mit der nötigen Dreiviertelmehrheit eine weitere Verfassungsreform beschlossen. Die Nationalversammlung als Verfassungsorgan wurde vollständig abgeschafft und ihre verbleibenden Kompetenzen auf den Legislativyuan übertragen. Mit der Abschaffung entspricht der Legislativ-Yuan nunmehr vollständig einem Parlament im herkömmlichen Sinne. Zudem wurde der Legislativyuan von 225 auf 113 Sitze verkleinert und die Wahlperiode von drei auf vier Jahre verlängert.

direkt. Weitere acht Abgeordnete werden mit den Stimmen von Auslandschinesen gewählt, und zwar nach den Stimmenanteilen der Parteien. Die übrigen 41 Mandate werden unter den Parteien 139 verteilt, die über $5 \%$ der Stimmen erlangten.

Siehe hierzu China aktuell 3/2000, S. 265. 
Die wohl größte Veränderung betrifft das Wahlrecht. Das bisherige Wahlrecht wurde abgeschafft. Ähnlich wie in Deutschland wird in Taiwan nun mit einer Erst- und Zweitstimme gewählt: die Erststimme für den Wahlkreiskandidaten, die Zweitstimme für Listenkandidaten der Parteien. Über die Zweitstimme ziehen jedoch von den insgesamt 113 Abgeordneten des Legislativyuan nur 23 Abgeordnete von Parteien in das Parlament ein, sofern diese die Fünfprozenthürde bewältigt haben. Die Wahlen 2008 finden dann erstmals nach dem neuen Wahlrecht und den entsprechenden Verfassungsänderungen statt ${ }^{140}$. Die Änderung des Wahlverfahrens wird die bisherigen eklatanten Mängel im Wahlrecht abstellen. Die Verlängerung der Mandate um ein Jahr und wird zur Stabilität künftiger Regierungen beitragen. Damit wird durch diese Verfassungsreform die Demokratie in Taiwan insgesamt gestärkt. Eine weitere wichtige Neuerung ist die Einführung von Plebisziten zu Verfassungsfragen. Voraussetzung ist jedoch, dass diese vom Parlament mit Dreiviertelmehrheit unterstïtzt werden $^{141}$.

\section{g. Bewertung}

Mit der Abschaffung der Nationalversammlung hat sich Taiwan eines Relikts aus der Bürgerkriegszeit entledigt, das aus verfassungsrechtlicher Sicht mit dem Aufbau eines demokratisch gewählten Parlaments, dem Legislativyuan, überflüssig wurde und kostspielig war $^{142}$. Das Verhältnis zwischen Präsident, Regierungschef und Legislativyuan ist jedoch weiterhin nicht eindeutig und austariert.

Trotz der unbestrittenen Erfolge der Verfassungsreform für die Demokratie muss das hieraus hervorgegangene politische System als problembehaftet bezeichnet werden ${ }^{143}$. Durch die schleichenden Änderungen des parlamentarischen Systems hin zu einem präsidialen System wurde ein systemimmanenter Konflikt geschaffen, der zu unklaren Kräfteverhältnissen führt, insbesondere zwischen Präsidenten und Premier. Die offensichtlichen Inkonsistenzen im Verfassungskonstrukt sind auf den „Stückwerkcharakter“ der Verfassungsreform zurückzuführen ${ }^{144}$.

140

Die Wahlen am 11.12.2004 verliefen daher noch nach dem alten Wahlrecht. Siehe zu dieser Wahl China Aktuell 12/2004, S. 1317.

141 Zu der Verfassungsänderung vom August 2004 siehe China Aktuell, 8/2004, S. 856ff.

142 Siehe hierzu China aktuell 4/2000, S. 386.

143 Tränkmann, Asien Nr. 67 (2000), S. 53, 57.

144 Zum Regierungssystem nach den Verfassungsänderungen siehe: Chen, Issues \& Studies Vol. 35 (1999) Nr. 9/10, S. 1-42; Cheng/Haggard, Political change in Taiwan (1992); Chu, Diss. Saarbrücken 1983; Chung, Free China Vol. 10 (1998) Nr. 8, S. 14-17; Gao, Free China Vol. 11 (1999) Nr.10, S. 7-21; Hsieh, Issues \& Studies Vol. 37 (2001) Nr. 1, S. 1-19; Liao/Tsai Issues \& Studies Vol. 37 (2001) Nr. 3, S. 1-31; Lumley, Asian Survey Vol. 25 (1976) Nr. 11, S. $1077-$ 
Seit der Verfassungsreform von $1997^{145}$ wird die Regierung durch den Premierminister geleitet, der ohne Zustimmung des Parlaments vom Präsidenten ernannt wird. Dennoch bleibt die Regierung gegenüber dem Parlament verantwortlich und ist von diesem auch abhängig, da das Parlament der Regierung das Vertrauen entziehen kann. In diesem Fall kann der Präsident das Parlament auflösen. Dieses System kann als semipräsidentiell bezeichnet werden ${ }^{146}$. Im Ergebnis ist die Regierung damit eingeklemmt zwischen Präsident und Parlament, was Spannungen garantiert, wenn Präsident und Parlamentsmehrheit nicht derselben Partei angehören ${ }^{147}$.

In der Ära Li Denghui kam es nicht zu Problemen, weil die von Präsident Li Denghui ernannten Premierminister GMD-Parteifreunde waren und deren Regierung im Legislativyuan von einer GMD-Mehrheit getragen wurde. Seit dem Wahlsieg Chen Shui-bians 2000 kam es jedoch wiederholt zu der Situation, dass der Präsident im Parlament keine Mehrheit fand $^{148}$. In solchen Fällen muss der Präsident einen von der Parlamentsmehrheit getragenen Premierminister ernennen, der nicht seiner eigenen Partei angehört, was insofern einer Kohabitation französischer Prägung ähnelt. Im Gegensatz zum französischen Präsidenten hat der Präsident der Republik China jedoch verfassungsrechtlich nicht die Befugnis, bei entgegenstehender Parlamentsmehrheit sofort das Parlament aufzulösen und Neuwahlen herbeizuführen $^{149}$. Ein solches Recht steht dem Präsidenten der Republik China nur im Falle einer negativ verlaufenen Vertrauensfrage zu. Dies führte zu einer schwierigen Regierungszeit mit wechselnden Premierministern ${ }^{150}$.

Die Verfassung dokumentierte auch nach allen Verfassungszusätzen nicht den Willen Taiwans als eigenständiger, von der Volksrepublik China unabhängiger Staat zu fungieren,

1095; McBeth, Asian Survey Vol. 40 (2000) Nr. 3/4, S. 251-268; Moon, Asian Survey Vol. 40 (1999) Nr. 1, S. 652-668; Schubert, Asien Nr. 79 (2001), S. 5-32; Schütte, China aktuell 5/2000, S. 508-516; Schütte, China aktuell 3/2000, S. 279-285; Schütte, China aktuell 7/1999, S. 696-704 (Teil 1) 8/1999, S. 872-833 (Teil 2); Cabestan, Asian Survey Vol. 40 (2000) Nr. 1, S. 172-180; $W u$, Asian Survey Vol. 39 (1999) Nr. 4, S. 565-587; Wu, Asian Survey Vol. 41 (1999) Nr. 1/2, S. 40-48; $W u$, Issues \& Studies Vol. 36 (2000) Nr. 9/10, S. 1-40.

Zur Verfassungsreform von 1986-1996 siehe insbesondere Chen, Issues and Studies Vol. 35 (1999) Nr. 5, S. 1-42.

$146 W u$, Issues and Studies Vol. 36 (2000) Nr. 5, S. 1, $5 \mathrm{ff}$.

147 Wu, Asian Survey Vol. 41 (2001) Nr. 1, S. 40, 45; Hsieh, Issues and Studies Vol. 37 (2000) Nr. 1, S. 1-19.

148

Zu den Wahlen vom 5. Dezember 1998 siehe Aretz, Freies China Vol. 12 (1999) Nr. 1/2, S. 2-9; Nieh, China aktuell 12/1998, S. 1334-1337; Wu, Asian Survey Vol. 39 (1999) Nr. 4, S. 565-587.

149 So konnte Präsident Mitterand 1981 und 1988 eine Kohabitation vermeiden. Dass ein solcher Vorgang auch riskant ist, zeigte der misslungene Versuch von Präsident Chirac im Jahre 1999.

Siehe zu den wechselnden Mehrheiten und Premierministern Wu, Asian Survey Vol. 41 (2001) Nr. 1, S. 40, 37f.; China aktuell 3/2000, S. 265; China aktuell 10/2000, S. 1165; China aktuell 3/2000, S. 264; China aktuell 5/2001, S. 488. 
da die gesamte Verfassungsstruktur weiterhin von dem Fortbestand eines Staates China ausgeht ${ }^{151}$.

\section{Die gegenwärtige Verfassungsdiskussion}

Das Ziel der chinesischen Einheit findet bei der Bevölkerung auf Taiwan nur bedingt Rückhalt. Die Insel Taiwan wird seit 1895 nicht mehr vom Festland regiert, der Großteil der Bevölkerung Taiwans hat die Zugehörigkeit Taiwans zum Festland daher nie erlebt. Im Gegensatz zur Bevölkerungsminderheit der 1949 mit der Nationalregierung nach Taiwan geflohenen Politiker, Beamten, Soldaten und deren Familien (den sogenannten "Festländern"), die aufgrund ihrer Verbindung zum Festland eine Wiedervereinigung wünschten und dieses Ziel an die ihnen nachfolgenden Generationen weitergaben, standen viele einheimische Taiwanesen dem offiziellen Ziel der Nationalregierung, der "Rückkehr auf das Festland", schlicht gleichgültig gegenüber. Sie wünschten sich nach Jahren der japanischen Fremdherrschaft (1895-1945) und der Diktatur der vom Festland geflohenen GMDRegierung einen unabhängigen taiwanesischen Staat ${ }^{152}$. Der Forderung dieser Bevölkerungsgruppe nach einer Unabhängigkeitserklärung konnte und wollte die GMD-Regierung während ihrer Regierungszeit jedoch angesichts eines befürchteten Militärschlags der Beijinger Regierung nicht nachgeben ${ }^{153}$.

An das Dogma der chinesischen Einheit hielten sich nach der Demokratisierung zunächst nicht alle zugelassenen Parteien ${ }^{154}$. Insbesondere die Demokratische Fortschrittspartei (DFP) kritisierte öffentlich das Festhalten an der chinesischen Einheit als reine Fiktion und forderte auf der Grundlage des Selbstbestimmungsrechtes ein Plebiszit der Bewohner Taiwans über die Unabhängigkeit und bei Zustimmung der Bevölkerung eine Unabhängigkeitserklärung und einen offiziellen Aufnahmeantrag als selbständiger Staat bei den Vereinten Nationen ${ }^{155}$. Wenngleich die DFP diese Forderungen mittlerweile aufgegeben hat,

Schubert, Rückblick auf eine Dekade des demokratischen Wandels- Reformen in Politik und Gesellschaft Taiwans, 1986-1994, S. 17, 27. Auch chinesische Verfassungsrechtler haben aus den Verfassungsänderungen keine Unabhängigkeitserklärung herausgelesen Chiang, Fordham International Law Journal Vol. 23 (2000), S. 959, 985. Aus der Wahl des Präsidenten alleine kann dies jedenfalls nicht gefolgert werden, so aber Breytenbach, SAJIL Vol. 2 (1994), S. 50, 53.

152 Trampedach, China auf dem Weg zur Wiedervereinigung?(1992), S. 33.

153 Trampedach, China auf dem Weg zur Wiedervereinigung??(1992), S. 46.

$154 \mathrm{Zu}$ den Positionen der wichtigsten taiwanesischen Parteien in der Chinafrage siehe Schubert, KAS-Auslandsinformationen, Vol. 12 (1997) Nr. 12, S. 30, 34ff.

155

Die DFP organisierte schon 1991 Demonstrationen für die Unabhängigkeit und eine eigene UNOMitgliedschaft Taiwans, AdG, 5.8.1991, 36031 A. Die DFP errichtete in New York sogar eigens ein Büro, dass das Selbstbestimmungsrecht der Taiwanesen sowie deren Recht auf eine eigene internationale Vertretung in der UNO und der Weltöffentlichkeit bekannt machen sollte. Durch 
kann eine starke „Taiwanisierung“ konstatiert werden: Taiwanesische Sprache und Kultur drängen in den Vordergrund zugunsten sich immer stärkere herausbildenden taiwanesischen Identität ${ }^{156}$. In diesem Zusammenhang ist auch die Diskussion um eine neue und eigene Verfassung zu betrachten. Eine neue Verfassung wäre zumindest aus rechtstechnischen Gründen zu begrüßen. Die Konstruktion der "Verfassungsergänzungen" durch die Verfassungszusätze von 1991, 1994, 1997 und 2004 ist rechtstechnisch nicht ideal. Das geltende Verfassungssystem, wiewohl reformiert, weist weiterhin Ungereimtheiten auf, die es als krisenanfällig erscheinen lassen ${ }^{157}$. Doch erscheint die von Präsident Chen Shui-bian bis 2006 angestrebte neue Verfassung ${ }^{158}$ eher unwahrscheinlich. Sie wäre als Symptom der Unabhängigkeit von Beijing mit dem Kriegsrisiko behaftet ${ }^{159}$. Zudem würde der einzig mögliche Weg eines Referendums eine Dreiviertelmehrheit im Legislativyuan voraussetzen, die jedenfalls derzeit unerreichbar ist.

Verfassungsrechtlich muss also auch in Zukunft an der Ein-China-Politik festgehalten werden. Viele der gegenwärtigen Verfassungsdiskussionen und Reformvorschläge bemühen sich daher um die Quadratur des Kreises: Die rechtliche Ausgestaltung einer modernen Demokratie im Rahmen einer Verfassung, die vor mehr als 60 Jahren nach längst überholten Verfassungslehren für einen nicht mehr existenten chinesischen Einheitsstaat entworfen wurde.

medienwirksame Aktionen erreichten sie dieses Ziel: Während einer UN-Vollversammlung kreiste über dem UN-Hauptgebäude ein Zeppelin mit dem Schriftzug "Taiwan in die Vereinten Nationen" und auf dem East River, nur wenige Meter vom UN-Gebäude entfernt, stand auf dem großen Segel eines Bootes dasselbe. Ebenso medienwirksam war der Versuch der DFP, den stets finanzklammen Vereinten Nationen einen Scheck als Geschenk der Bürger Taiwans zu überreichen. Aufgrund des Veto der Regierung in Beijing musste dieser Scheck zurückgereicht werden. Nicht zuletzt durch diese Aktionen der DFP wurde das chinesische Vertretungsproblem in der Weltöffentlichkeit verstärkt wahrgenommen. Zu dieser Auffassung siehe die Ausführungen der DFPPolitiker Chang und Lim im Journal of International Law and Foreign Affairs Vol. 1 (1996) Nr. 2, S. 393, 394ff.

Zur Entwicklung einer taiwanesischen Identität siehe: Schubert, Asien Nr. 79 (2001), S. 5, 7ff.; Schneider, Asien Nr. 62 (1997), S. 46-67; Schubert, Internationale Politik Vol. 3 (1998), Nr. 267274; Chao, Issues \& Studies Vol. 30 (1994) Nr. 4, S. 1-13; Liu/Ho, Issues \& Studies Vol. 35 (1999) Nr. 5/6, S. 1-34; Weth, Diss. Würzburg 1978; Schubert, China Perspectives Nr. 23 (1999), S. 54-64; Corcuff, China Perspectives Nr. 28 (2000), S. 71-81; Inghai, China Perspectives Nr. 28 (2000), S. 82-88; Allio, China Perspectives Nr. 28 (2000), S. 43-50; Chang, China Perspectives Nr. 28 (2000), S. 51-65.

$W u$, Streitbare Demokratie, S. 129.

158 Im September 2003 forderte Präsident Chen Shui-ban auf einer Parteiveranstaltung eine neue 159 Verfassung für Taiwan, China Aktuell 9/2003, S. 1093.

China Aktuell 12/2003, S. 1460, 1461. 


\section{Ausblick}

Im März 2004 wurde Präsident Chen Shui-bian wieder gewählt, das von ihm initiierte Referendum über die Verteidigung Taiwans hingegen scheiterte ${ }^{160}$. Auch im Vorfeld des letztendlich gescheiterten "Defensiv Referendums" von Präsident Chen Shui-bian gab es ernsthafte Drohungen aus Beijing und harsche Kritik aus dem Inland wie aus dem Ausland. Letztendlich trug Chen Shui-bian hiermit zu dem Scheitern bei den Wahlen zum Legislativyuan im Dezember 2004 bei, was ihn letztendlich auch das Amt des DFP-Vorsitzenden kostete $^{161}$. Die Schritte Chens müssen vor dem Hintergrund des gleichwohl starken Wunsches vieler Taiwanesen nach Unabhängigkeit gesehen werden. So demonstrierten am 6. September 2003100.000 Bürgerinnen und Bürger für die Umbenennung von Republik China zu Taiwan ${ }^{162}$.

Die heute gültige hohe Hürde für Referenden ist für den Frieden in der Taiwanstraße sicherlich gut, denn eine Volksabstimmung in Taiwan über die Zukunft des Landes hatte Beijing immer schon als Kriegsgrund betrachtet. Ein Schritt in Richtung Entspannung war sicherlich der Abzug von großen Teilen der taiwanesischen Truppen von den festlandnahen Inseln (Jinmen und Kinmen) ${ }^{163}$. Chen Shui-bian hat sich in Beijing dennoch nicht beliebt gemacht. Im Gegenteil, seine Pläne zur Einführung einer neuen Verfassung, zu Aufnahmeanträgen bei den Vereinten Nationen unter dem Namen "Taiwan", das "Defensiv Referendum" sind von Beijing stark kritisiert worden. In diesem - auch zeitlichen - Kontext muss man das im März 2005 von Beijing erlassene Antisezessions-Gesetz sehen.

Regierung und Opposition in Taiwan sind in der Frage des taiwanesischen Selbstverständnisses weiter uneins. Es können prinzipiell drei Richtungen und damit drei Fragen aufgezeigt werden: 1. Soll Taiwan mit der Volksrepublik wiedervereint werden und wenn ja, wann und wie? Soll Taiwan den Status quo erhalten und wenn ja, was muss man dafür noch tun? 3. Soll Taiwan sich unabhängig erklären und wann ja, wann und wie? In regelmäßigen Umfragen wird die Bevölkerung hierzu befragt. Die stark zunehmende Taiwanisierung auf der einen Seite und die als ernster empfundenen Bedrohung durch die Volksrepublik auf der anderen Seite lässt die prozentuale Anzahl der Befürworter einer Wiedervereinigung langsam aber sicher sinken ${ }^{164}$.

\footnotetext{
160

161

China Aktuell 3/2004, S. 275.

162

China Aktuell 12/2004, S. 1323; China Aktuell 1/2005, S. 78.

163

164

China Aktuell 9/2003, S. 1093.

China Aktuell 10/2004, S. 1108.

China Aktuel12/2003, S. 1464, 1471f.
} 
Die Verfassungsdebatte in Taiwan geht daher weiter. Wenn nicht ein Krieg mit der Volksrepublik riskiert werden soll, muss auch in Zukunft verfassungsrechtlich an der Ein-ChinaPolitik und der Verfassung von 1947 festgehalten werden. Es wird daher wohl auch in der Zukunft nur zu Veränderungen durch Verfassungszusätze kommen. 